\title{
European Survey Data on Attitudes to Equality and Human Rights
}

Technical Paper

Éamonn Fahey, Doireann O'Brien Helen Russell, Frances McGinnity

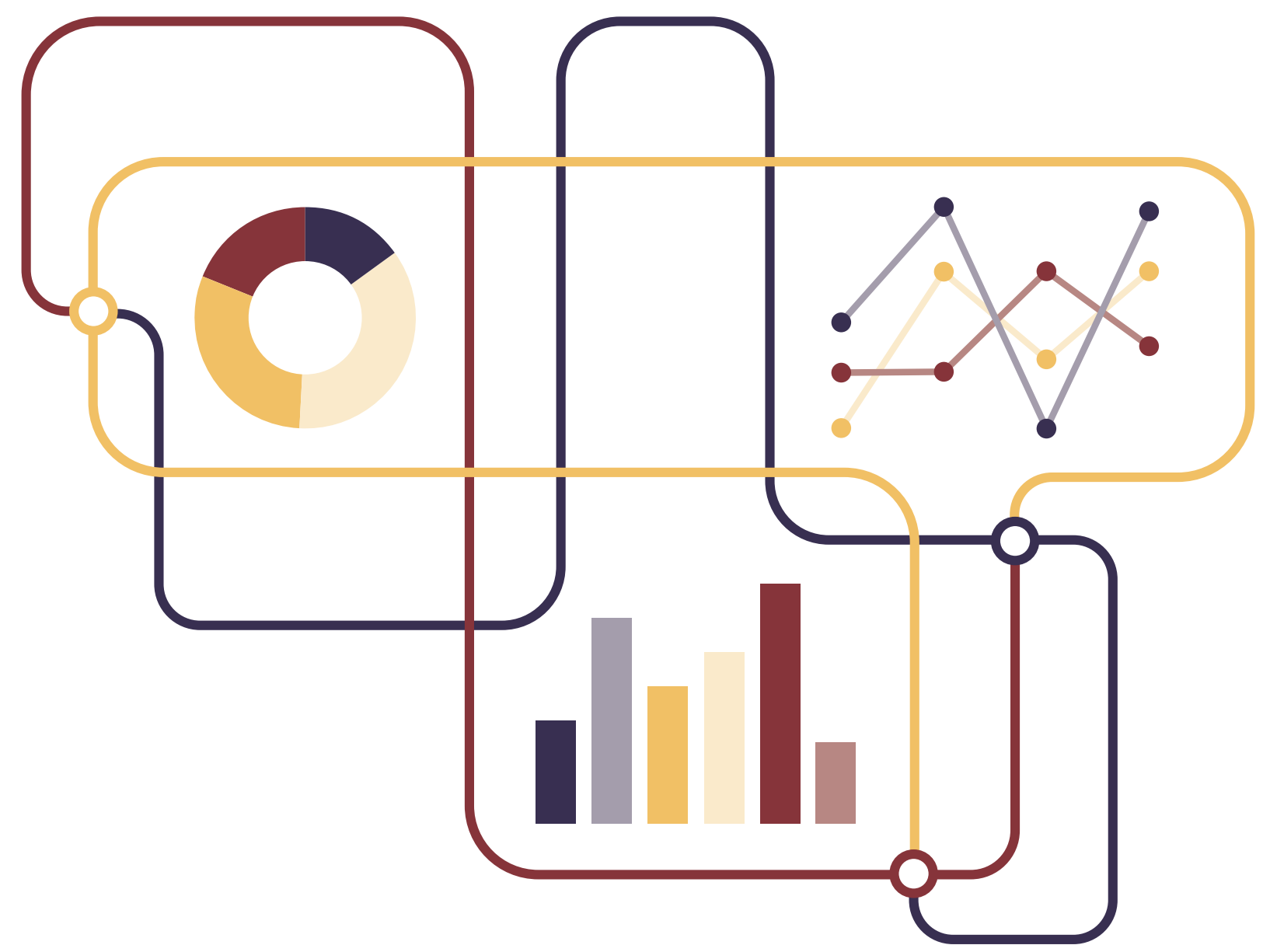

Coimisiún na hÉireann um Chearta an Duine agus Comhionannas

Irish Human Rights and Equality Commission

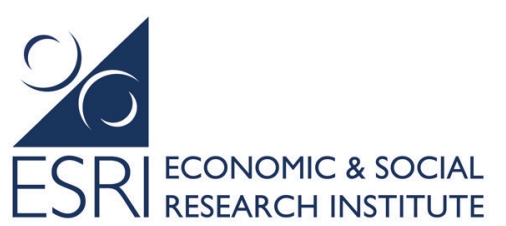




\section{European Survey Data on Attitudes to Equality and Human Rights}

Technical Paper

Éamonn Fahey, Doireann O'Brien Helen Russell, Frances McGinnity 
This report was written by Eamonn Fahey, Doireann O'Brien, Helen Russell and Frances McGinnity. It was prepared for the Irish Human Rights and Equality Commission by the Economic and Social Research Institute as part of the Research Programme on Human Rights and Equality. The report has been peer-reviewed prior to publication. The views expressed in this report are those of the authors and do not necessarily represent those of the Economic and Social Research Institute or the Irish Human Rights and Equality Commission.

The Irish Human Rights and Equality Commission was established under statute on 1 November 2014 to protect and promote human rights and equality in Ireland, to promote a culture of respect for human rights, equality and intercultural understanding, to promote understanding and awareness of the importance of human rights and equality, and to work towards the elimination of human rights abuses and discrimination.

The Economic and Social Research Institute (ESRI) is an independent research institute working towards a vision of 'Informed policy for a better Ireland'. The ESRI seeks to support sustainable economic growth and social progress in Ireland by providing a robust knowledge base capable of providing effective solutions to public policy challenges.

Available to download from www.esri.ie and www.ihrec.ie

(C) 2019. Copyright is held jointly by the Economic and Social Research Institute and the Irish Human Rights and Equality Commission.

Irish Human Rights and Equality Commission, 16-22 Green Street, Dublin 7.

The Economic and Social Research Institute, Whitaker Square, Sir John Rogerson's Quay, Dublin 2

ISBN 978-0-9957296-3-6

DOI https://doi.org/10.26504/sustat83.pdf

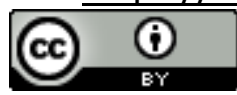

This Open Access work is licensed under a Creative Commons Attribution 4.0 International License (https://creativecommons.org/licenses/by/4.0/), which permits unrestricted use, distribution, and reproduction in any medium, provided the original work is properly credited. 


\section{THE AUTHORS}

Éamonn Fahey was a Research Assistant at the Economic and Social Research Institute (ESRI). Helen Russell is a Research Professor at the ESRI and an Adjunct Professor at Trinity College Dublin (TCD). Doireann O'Brien was on a research internship at the ESRI at the time of writing. Frances McGinnity is an Associate Research Professor at the ESRI and an Adjunct Professor at Trinity College Dublin (TCD).

\section{ACKNOWLEDGEMENTS}

The authors gratefully acknowledge the funding received from the Irish Human Rights and Equality Commission (IHREC) to support the research programme. We would like to thank the members of the programme steering group, Tania Burchardt (London School of Economics), Cliona Doherty (IHREC), Iris Elliot (IHREC) and Cian Finn (IHREC) for their constructive comments on the research. We have also benefited from the comments of an internal ESRI reviewer and Emer Smyth (ESRI) as editor. We would also like to extend our thanks to Sarah Burns (ESRI) and Anna de Courcy (WordFocus) for their work in preparing this report for publication. 


\section{TABLE OF CONTENTS}

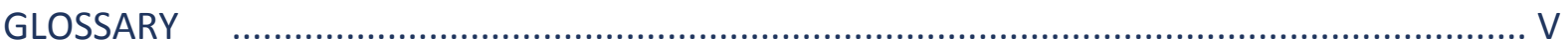

ABSTRACT

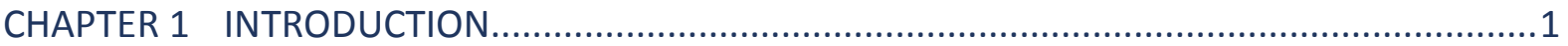

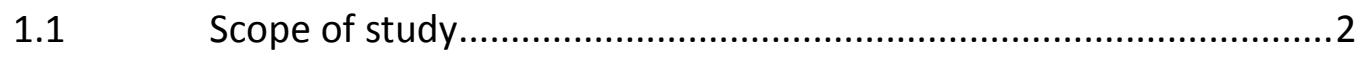

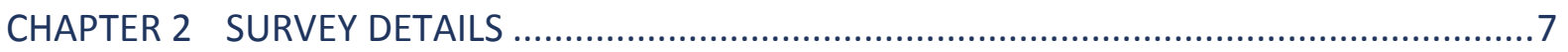

2.1 Strengths and weaknesses of attitudinal survey data.....................7

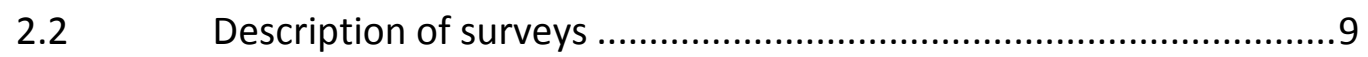

$2.3 \quad$ Frequency of surveys .......................................................... 18

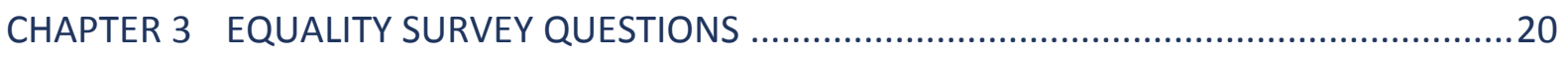

3.1 Race, ethnicity, nationality and religion .......................................21

3.2 Gender, civil and family status and sexual orientation ..................28

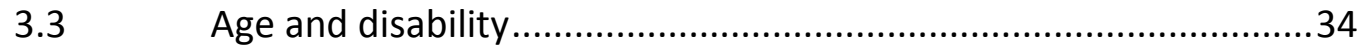

3.4 Attitudes towards social welfare and equality in general ...............38

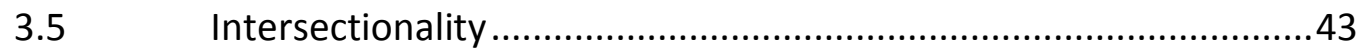

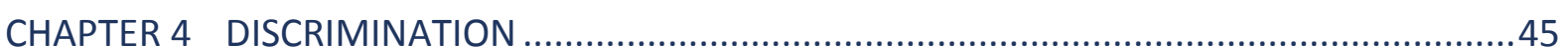

Experiences of discrimination.................................................... 46

4.2 Witnessing discrimination ....................................................51

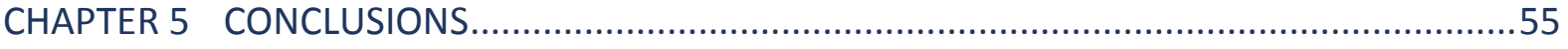

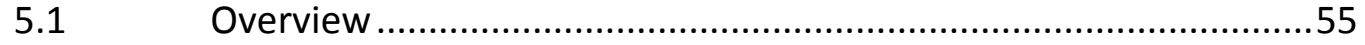

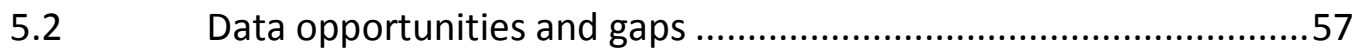

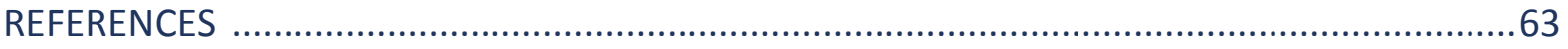

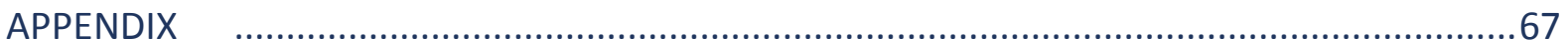




\section{LIST OF TABLES}

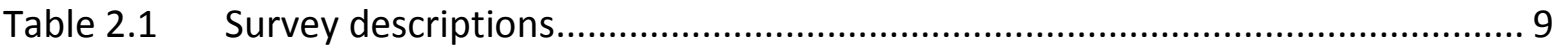

Table 3.1 Most frequently asked questions: race, ethnicity and nationality.................. 26

Table 3.2 Most frequently asked questions: miscellaneous equality ............................ 44

Table A.1 Topic categories used in question-bank spreadsheet .................................67

\section{LIST OF FIGURES}

Figure 2.1

Figure 2.2

Figure 3.1

Figure 3.2

Figure 3.3

Figure 3.4

Figure 3.5

Figure 3.6

Figure 3.7

Figure 3.8

Figure 3.9

Figure 3.10

Figure 4.1

Figure 4.2

Figure 4.3
Total selected questions by survey, 2000-2018. 18

Percentage of questions per year from each survey 19

Question count per target group, all surveys 2000-2018 21

Race, ethnicity and nationality attitudes question count, all surveys 24

Religion attitudes question count, all surveys 27

Gender attitudes question count, all surveys 30

Civil and family status attitudes question count, all surveys 32

Sexual orientation attitudes question count, all surveys. 34

Age attitudes question count, all surveys .36

Disability attitudes question count, all surveys . 38

Social welfare attitudes question count, all surveys. 40

Miscellaneous equality and human rights question count, all surveys ... 42

Question count per target group, all surveys 2000-2018 46

Discrimination experience question count, all surveys 48

Discrimination witness question count, all surveys 52 


\section{GLOSSARY}

CSO

ESS

EVS

FRA

IHREC

ISSP
Central Statistics Office

European Social Survey

European Values Study

European Union Fundamental Rights Agency

Irish Human Rights and Equality Commission

International Social Survey Programme

Multi-stage probability/stratified sampling design

The first stage of this design involves a random sample of 'primary sampling units' being used (in Ireland usually the Census small areas), and then within those units a random list of households are sampled. These samples can be stratified so their makeup is proportional to the makeup of the country (e.g. including the same proportion of men and women in the sample as the national proportion).

Sample weighting Sampling weights are used when the proportion of certain groups in a sample is not the same as the proportion of that group in the whole population. Weights are applied to over- or under-represented groups to increase the accuracy of survey estimates.

Population size weighting

These weights are used when countries have similar sample sizes regardless of the size of their overall population. The weights ensure countries are represented proportionately for their population size within data on a group of countries.

Two-stage clustered stratified design:

The first stage of this design involves a random sample of 'primary sampling units' being used (in Ireland usually the Census small areas), and then within those units a random list of households are sampled. These samples can be stratified so their makeup is proportional to the makeup of the country (e.g. including the same proportion of men and women in the sample as the national proportion). The clustering is done by splitting the survey population into smaller geographical areas, and then only including some of these clusters in the final stage sampling of random households. 


\section{ABSTRACT}

This report maps the data available on attitudes to equality and human rights issues in Ireland collected in European surveys over the period 2000 to 2018. These surveys provide a rich source of evidence for researchers and policymakers in Ireland. We identify a total of 1,509 relevant questions from a search of over 125 attitude surveys. These questions are categorised according to the groups and topics addressed. We find that attitudes towards minority ethnic/nationality groups, gender/gender roles and social welfare recipients are the most widely covered. Questions on attitudes towards religious minorities, age groups/ageism, family status, disability and sexual orientation are much less common. Moreover, while the frequency of attitude questions relating to sexual orientation has increased over the period, questions on age and disability groups have declined. Within these equality groups the surveys cover a range of topics including social distance, social contact, tolerance and policy preferences.

Questions on experiencing or witnessing discrimination are also regularly included in European questionnaires: a total of 359 such questions are identified over the period studied. Again, coverage is greatest for gender and ethnicity/nationality groups; however, the third most common set of questions concerns discrimination on the basis of sexual orientation. We identify a data gap in discrimination on the grounds of social welfare receipt or social class. Intersectionality - that is the way in which the combination of equality characteristics e.g. gender and ethnicity, may be associated with differential treatment or in this case viewed differently by others - is rarely covered in the surveys we assessed.

The research highlights the availability of data that can be analysed to provide insights into where policy efforts to reduce prejudice and discrimination might best be targeted. Analysis of such questions can also highlight which policies are more likely to encounter public resistance or support. The report also discusses some of the strengths and limitations of attitudinal surveys for addressing equality and human rights issues and draws out some lessons for questionnaire design. 


\section{CHAPTER 1}

\section{Introduction}

This report seeks to map the availability of Irish data on attitudes to equality and human rights issues from European surveys. Through an intensive manual search of questionnaires, technical reports and online question banks from over 125 surveys, the research identifies 1,509 attitudinal questions on equality and human rights. These questions were subsequently categorised in terms of their subject matter and an original database of questions was produced. The question database was then analysed to identify themes, trends and gaps. The questions relate to surveys carried out by five international organisations over the period 2000 to 2018 . The report is written to inform the development of future research and data work by the Irish Human Rights and Equality Commission, and to identify sources of information which could be harnessed to develop evidence-based policy and by researchers more generally.

The report contributes to improving the collection and use of equality data advocated in the guidelines of the EU High Level Group on Non-Discrimination, Equality and Diversity (European Commission, 2018). The term Equality Data is defined as

any piece of information that is useful for the purposes of describing and analysing the state of equality. The information may be quantitative or qualitative in nature. It could include aggregate data that reflect inequalities or their causes or effects in society (European Commission, 2018 p.4).

In particular, the research undertaken can help to contribute to a number of areas identified in the guidelines including mapping existing sources of equality data and identifying data gaps, and 'facilitating the effective use of equality data' by highlighting where comparative high quality information on attitudes to equality groups and human rights can be found.

Carefully designed survey data on societal attitudes towards groups protected under equality legislation and towards rights protected under international agreements provide 
important information on the state of equality and can potentially provide insights into the processes that produce and reproduce inequalities. Such data can provide, for example, information on the perceived social distance between groups and the extent to which respondents are tolerant of diversity or the prevalence of stereotypical views (for example McGinnity et al., 2018a). Social attitudes data can also shed light on policy interventions that are likely to garner public support and resistance and can highlight issues on which the public are misinformed or lack information. Such data complement other sources of information on inequalities and discrimination such as data on outcomes, experimental research, administrative data, qualitative research, and legal judgements/caseloads (Bond et al., 2010; EU Commission, 2018).

\subsection{SCOPE OF STUDY}

Five criteria were used to determine inclusion in the analysis. The survey needed to be international in scope (covering people in multiple EU countries), include Ireland in at least one round, be available for analysis by researchers or policymakers, ${ }^{1}$ be representative of the population from which they are drawn and ask questions relevant to assessing public attitudes to equality and human rights issues. These criteria yielded five sets of surveys: the Eurobarometer, the European Social Survey (ESS), the European Values Study (EVS), the International Social Survey Programme (ISSP) and the surveys of the EU Fundamental Rights Agency (FRA). The surveys are described in the following chapter.

The report does not cover surveys, or survey questions, on equality outcomes; for example surveys on the economic or social circumstances of different groups in society and their access to resources or services. Therefore surveys such as the EU Statistics on Income and Living Conditions (EU-SILC) and the EU Labour Force Survey (EU LFS) are not included as they do not include questions on attitudes. ${ }^{2}$

\footnotetext{
1 The surveys identified have different application procedures and data usage rules but in principle are available for further data analysis. This criterion excludes surveys run on behalf of private organisations. 2 However, we acknowledge the value of this kind of data, and these surveys have been used extensively in previous ESRI and IHREC research.
} 
The bulk of the analysis is focused on questions fielded to the general public about their attitudes towards equality groups, by which we mean the ten groups protected in Irish legislation (Employment Equality Acts 1998-2015; Equal Status Acts 2000-2015). ${ }^{3}$ These are:

- $\quad$ Race, ethnicity, nationality;

- Membership of the Traveller Community;

- Religion;

- $\quad$ Gender;

- Sexual orientation;

- $\quad$ Family status;

- $\quad$ Civil status;

- $\quad$ Age;

- Disability;

- $\quad$ Receipt of social welfare or housing assistance (discrimination in relation to housing only).

Because we are looking at international surveys, and members of the Traveller community are highly concentrated in Ireland, we did not expect to find many questions relating to attitudes towards this group, but any questions for this group or Roma are included with those on race, ethnicity and nationality. For ease of presentation, we also group questions on family and civil status. The final category, receipt of social welfare, covers attitudes to welfare recipients as well as attitudes to the role of social welfare. Under this heading we also included questions on attitudes to income inequalities and views on the causes of inequality and poverty. This is done in accordance with IHREC's strategic prioritisation of socio-economic rights ${ }^{4}$ (IHREC, 2019), and its call for the recognition of socio-economic status as a protected characteristic under equality legislation (IHREC, 2017).

These equality groups overlap considerably with the grounds of discrimination recognised in human rights law, which includes sex, age, disability, civil status, ethnicity, migration or

In the literature these are also referred to as 'protected grounds' or 'protected groups'.

Socio-economic rights refer to the economic, social and cultural rights protected under international human rights law such the right to an adequate standard of living, the right to decent work, the right to physical and mental health, and the right to education among others. 
displacement status, religion, income, sexual orientation and gender identity (UN, 2018). We also include survey questions that ask directly about attitudes to human rights. These provide information on societal support for different aspects of human rights and policy measures to protect those rights.

Within this division by equality group, we attempted to categorise questions in the database into meaningful themes. Although they are somewhat arbitrary, they help the reader to understand what kind of questions appear most frequently in the surveys over this time period. These are discussed in detail in Chapter 3.

Throughout, we have attempted to limit these questions to survey items that tap into an attitude. Attitudinal questions are by definition subjective in that they involve an evaluative element or normative opinion (how things should be) rather than recording objective characteristics, outcomes or facts (how things are). Eagly and Chaiken (1993) define attitudes as 'a psychological tendency that is expressed by evaluating a particular entity with some degree of favor or disfavor'. Attitudes generally cannot be verified or falsified by external criteria.

When searching through questionnaires and question banks, it proved difficult to make a distinction between normative and factual questions in some cases. Many questions are clearly normative, such as those that include the word 'should'. For example the 2015 Eurobarometer simply asks if the respondent agrees with the statement that Ireland 'should help refugees'. Other questions are clearly normative despite not including the word 'should', e.g. 'How comfortable would you be having a disabled person as a neighbour'. 5 However, it is difficult to decide if some questions are normative or factual as some questions may appear to ask about a factual issue but the answers are likely to embody evaluations. For example, the 2017 Eurobarometer asks respondents 'how does the media portray immigrants?' This is at first sight a simple, factual question, but the response options are 'too positively, objectively or too negatively'. If the respondent says 'too positively', this could be interpreted as anti-immigrant sentiment. Discussion and consensus among the members of the research team was used to resolve these issues. Similarly, some 
questions tap into beliefs that are verifiable, for example, the belief that migration has a positive or negative effect on the economy is testable with objective economic data. The belief that the association is positive could reflect a favourable attitude to migrants or may be based on knowledge of economic facts. We have included questions that assess these beliefs since they potentially reveal social attitudes.

In addition to questions on the attitudes of the general public towards equality groups and to equality and human rights in general, we separately consider questions which ask whether participants have ever experienced discrimination or witnessed discrimination. IHREC's research agenda in recent years has drawn heavily on questions on the experience of discrimination fielded in the Central Statistics Office (CSO)'s domestic survey on equality, the Equality module of the Labour Force Survey. This survey relies on a very large sample size to capture enough respondents of minority groups while maintaining representativeness of the population as a whole. ${ }^{6}$ With the exception of the FRA surveys which target minority groups rather than the overall population, the surveys included in this report likely only capture very small samples of minority ethnic groups, people with disabilities and the LGBT community. This is because their total Irish sample in each round is between 500 and 2,000 depending on the survey. Disaggregation of discrimination experience also requires that the data include the relevant characteristics (such as the respondents' sexual orientation). Accurately assessing the experience of discrimination among minority populations in a general survey requires much larger samples. ${ }^{7}$ This might be achieved through booster samples (over-sampling) of particular groups or targeted samples of geographic areas where there is a higher concentration of the group of interest (UN, 2018). Within the current set of surveys, questions that ask whether the respondent has ever witnessed discrimination against other people may allow researchers to estimate the prevalence of discrimination despite these small samples.

The next chapter of this report discusses in detail the strengths and weaknesses of surveys in attitudinal equality research, before providing an overview of the Eurobarometer, ESS,

\footnotetext{
6 The largest of these samples, taken in 2004, captured 24,610 respondents but sample size has fallen over time.

7 This point also applies to the analysis of outcomes across equality groups, but this is not the focus of the current exercise.
} 
EVS, ISSP and FRA surveys. Chapter 3 examines the distribution of questions on attitudes to equality groups and equality in general across these surveys and over time. It portrays the frequency of various types of questions graphically, and provides examples of typical questions asked about each equality group. Chapter 4 carries out similar exercises for questions on the experience and witnessing of discrimination. Chapter 5 draws conclusions on data opportunities and gaps. 


\section{CHAPTER 2}

\section{Survey details}

This chapter presents a broad overview of the strengths and weaknesses of survey data for investigating attitudes towards equality and human rights issues, and discusses the characteristics of the surveys covered in this report. We cover five sets of surveys carried out between 2000 and 2018. These are the Eurobarometer, the European Social Survey, the International Social Survey Programme, the European Values Study and the surveys of the Fundamental Rights Agency. All five surveys are repeated and cross-national, include Ireland in at least one wave and provide significant information on discrimination and attitudes to equality and to human rights. In total just over 1,500 relevant questions are compiled in a database which is available on the ESRI and IHREC websites (https://www.esri.ie/publications/european-survey-data-on-attitudes-to-equality-groupsand-human-rights and https://www.ihrec.ie/documents/european-survey-data-onattitudes/) with this figure including questions that have been asked repeatedly. We provide a broad overview of these questions in the following chapters.

\subsection{STRENGTHS AND WEAKNESSES OF ATTITUDINAL SURVEY DATA}

Survey data provide an invaluable resource for understanding people's attitudes towards equality and human rights and their experiences of discrimination in daily life. When conducted correctly, surveys can be a remarkably efficient and cost effective way of gauging the views of the public. High quality surveys are representative of the population, flexible, can track attitudes and experiences over time and are ideal for cross-national research.

The surveys that we include here are of a relatively high standard, and do not rely on convenience sampling. They generally attempt to minimise non-response in a systematic fashion, and validate the characteristics of the sample with external sources such as population Censuses or administrative data that have a greater chance of achieving representativeness. 
However, survey-based social science research is also challenging. Behavioural studies have found that people's responses to questions are remarkably sensitive to seemingly unrelated factors. Key among these are priming and framing effects (Zaller and Feldman, 1992). Priming effects refer to a situation where a participant's response is influenced by an earlier question in the survey. Framing effects are biases introduced by the way in which the question is asked. Ideally, questions should be posed in a neutral fashion to avoid inadvertently encouraging the respondent to choose one option over the other (Simmons, 2001). Another challenge for surveys which tackle sensitive issues such as equality, discrimination and human rights is social desirability bias. A growing body of scholarship suggests that many survey respondents are reluctant to reveal socially undesirable responses, particularly when participating in a face-to-face interview (e.g. Heerwegh, 2009; Brownback and Novotny, 2018).

Survey research is only one of a wide variety of methods for collecting equality data (Bond et al., 2010; EU Commission, 2018). Triangulating the results of survey research with other information sources such as administrative data, qualitative research, legal caseload data, and experimental research is important for building a comprehensive and robust evidence base. 


\subsection{DESCRIPTION OF SURVEYS}

\section{TABLE 2.1 SURVEY DESCRIPTIONS}

\begin{tabular}{|c|c|c|c|c|}
\hline Title & Frequency & $\begin{array}{l}\text { Sample size in } \\
\text { Ireland* }\end{array}$ & Groups covered & Availability of data files \\
\hline $\begin{array}{l}\text { Euro- } \\
\text { barometer }\end{array}$ & $\begin{array}{l}\text { Main modules } \\
\text { twice a year, } \\
\text { additional special } \\
\text { modules usually } \\
\text { four times a year }\end{array}$ & $\sim 1,000$ & $\begin{array}{l}\text { Samples from } \\
\text { residents over the age } \\
\text { of } 15\end{array}$ & $\begin{array}{l}\text { Free to download in SPSS or } \\
\text { STATA format from the } \\
\text { Leibniz Institute for the } \\
\text { Social Sciences website } \\
\text { (GESIS) }\end{array}$ \\
\hline ESS & Every two years & $\begin{array}{l}\text { Fluctuates } \\
\text { between } 1,800 \\
\text { and about } 2,800 \\
\text { depending on } \\
\text { the year }\end{array}$ & $\begin{array}{l}\text { Samples from } \\
\text { residents over the age } \\
\text { of } 15\end{array}$ & $\begin{array}{l}\text { Free to download from ESS } \\
\text { website }\end{array}$ \\
\hline ISSP & $\begin{array}{l}\text { Survey released } \\
\text { every year, but not } \\
\text { always fielded in } \\
\text { the same year. } \\
\text { Stopped being run } \\
\text { in Ireland in } 2013 \text {. }\end{array}$ & $\begin{array}{l}\text { Usually around } \\
1,000 \text {, although } \\
\text { the } 2009 \text { and } \\
2008 \text { waves had } \\
\text { sample sizes of } \\
\sim 2,000\end{array}$ & $\begin{array}{l}\text { Samples from } \\
\text { residents }\end{array}$ & $\begin{array}{l}\text { Free to download in SPSS or } \\
\text { STATA format from the } \\
\text { Leibniz Institute for the } \\
\text { Social Sciences website } \\
\text { (GESIS) }\end{array}$ \\
\hline EVS & $\begin{array}{l}\text { Every nine years. } \\
\text { The latest wave } \\
\text { was not run in } \\
\text { Ireland }\end{array}$ & $\sim 1,000$ & $\begin{array}{l}\text { Samples from } \\
\text { residents over the age } \\
\text { of } 18\end{array}$ & $\begin{array}{l}\text { Free to download in SPSS or } \\
\text { STATA format from the } \\
\text { Leibniz Institute for the } \\
\text { Social Sciences website } \\
\text { (GESIS) }\end{array}$ \\
\hline FRA & $\begin{array}{l}\text { Irregular. } \\
\text { - Minorities and } \\
\text { Discrimination } \\
\text { Survey: } 2016 \\
\text { - Violence Against } \\
\text { Women Survey: } \\
2012 \\
\text { - LGBT Survey: } \\
\text { 2012, second } \\
\text { LGBT survey was } \\
\text { in } 2019 \text { (not } \\
\text { covered in this } \\
\text { report) }\end{array}$ & $\begin{array}{l}\text { - Minorities } \\
\text { and } \\
\text { Discriminatio } \\
\text { n Survey: } 425 \\
\text { - Violence } \\
\text { Against } \\
\text { Women } \\
\text { Survey: 1,567 } \\
\text { - LGBT Survey: } \\
\text { 1,625 }\end{array}$ & $\begin{array}{l}\text { - Minorities and } \\
\text { Discrimination } \\
\text { Survey: Samples } \\
\text { from immigrants } \\
\text { from Sub-Saharan } \\
\text { Africa or their } \\
\text { descendants. } \\
\text { - Violence Against } \\
\text { Women Survey: } \\
\text { Samples from } \\
\text { women aged } 18 \text { to } \\
74 . \\
\text { - LGBT Survey: } \\
\text { Samples from LGBT } \\
\text { people over the age } \\
\text { of } 18\end{array}$ & $\begin{array}{l}\text { - Minorities and } \\
\text { Discrimination Survey: } \\
\text { expected to be available } \\
\text { by end of } 2019 \\
\text { - Violence Against Women } \\
\text { Survey: Available from the } \\
\text { UK Data Service, upon } \\
\text { registration of an account } \\
\text { and filling in of a special } \\
\text { license form } \\
\text { - LGBT Survey: Available } \\
\text { from the UK Data Service, } \\
\text { upon registration of an } \\
\text { account and filling in of a } \\
\text { special license form }\end{array}$ \\
\hline
\end{tabular}

Source: ESRI Time-Use Survey (2005) and European Quality of Life Survey (2007-2016).

Note * With a nationally representative sample of 1,000 , any groups that comprise less than 5 per cent of the overall population will yield insufficient cases for statistical analysis ( $<50$ cases). This will be exacerbated if the groups are hard to reach, perhaps due to disadvantage. 


\subsubsection{Eurobarometer}

Eurobarometer was first conducted in 1974 and is run within the European Union Member States on behalf of the European Commission. ${ }^{8}$ Its main purpose initially was to inform the European Commission about attitudes towards European affairs and the European Union. It now also includes modules on topics capturing other attitudes and behaviours of the European public.

The standard Eurobarometer is conducted twice a year in autumn and spring. Special modules on different subjects are conducted at other times throughout the year, while smaller Flash Eurobarometers are conducted intermittently. This results in at least six surveys in most years. Eurobarometer is conducted in all European Union Member States as well as in other European countries in some years. It is a repeated cross-sectional survey, drawing new independent samples in every country for each time point. The typical sample size per country is 1,000 interviews, although this is smaller or larger for some particularly small and large countries. In Ireland the sample size is usually around 1,000. The Flash Eurobarometer surveys approximately 500 respondents per country.

A multi-stage random probability sampling design is used. Primary sampling units (PSUs) are chosen to ensure that the sample matches the population in terms of metropolitan, urban and rural location. Within PSUs, households are typically selected for interview by random walk. Under this procedure, interviewers are assigned a starting point within the PSU and are given instructions on how to select houses. Data collection is typically carried out by computer assisted face-to-face interviews. The smaller Flash Eurobarometers are telephone based surveys.

Eurobarometer data are openly available and free to download in SPSS or STATA format from the Leibniz Institute for the Social Sciences website (GESIS), though users have to 
register an email address and state a purpose for accessing the data (e.g. scientific research, professional or commercial purposes). ${ }^{9}$

An extensive set of weighting variables is available in Eurobarometer to ensure that the sample is appropriate for national and comparative analyses. There are two kinds of weights; post-stratification sample weighting and population size weighting. Statistics provided by Eurostat or by National Survey Research Institutes inform the European Commission of the true distribution of the population of each country in terms of age, sex, region and size of locality. The post-stratification weights ensure that the Eurobarometer samples approximate these distributions by adjusting for non-response. Population size weighting accounts for the fact that although countries in Europe vary substantially in terms of population, the national sample size is similar across countries. In other words, small countries are over sampled relative to their population size, and large countries are under sampled.

\subsubsection{European Social Survey}

The European Social Survey (ESS) was founded in 2001, with the first round of surveys being conducted in $2002 .{ }^{10}$ The ESS is a high quality, academically driven survey established to investigate changes over time in attitudes and behaviour in Europe. It consists of a core section that is included in every round to measure topics considered to be of particular interest and to measure background variables, as well as two special modules which change every round. ${ }^{11}$ These modules are occasionally repeated to allow for comparison over time.

The ESS is conducted every two years, and 38 countries have taken part in at least one round. Fifteen countries, including Ireland, have taken part in every round. National funding is required to run the survey in individual countries and to contribute to the central costs.

9 A bibliography of publications produced using the Eurobarometer data is maintained by GESIS https://www.gesis.org/fileadmin/upload/dienstleistung/daten/umfragedaten/eurobarometer/eb_bibliog raphy/EB_Bibliography.pdf [last accessed 30 October 2019].

10 All information on the ESS was sourced from the European Social Survey website. The link can be found in the References section of this report.

11 The special module topics for the ESS are selected through an open competition. Groups of academics, which must span at least three countries, are invited to submit a proposal for a special module. The proposals are evaluated by the Scientific Advisory Board of the ESS. 
Funding for the Irish element of the survey comes from the Irish Research Council. We analyse questions across nine rounds of the survey, from 2002 to 2018.

The ESS is a cross-sectional survey. In each participant country, the samples aim to be representative of all residents of the country older than 15 years of age. The central ESS body oversees the harmonisation of methods between countries (see: www.europeansocialsurvey.org/methodology/ess_methodology/data_collection.html) . Individuals must be chosen randomly, usually from a sampling frame such as a population register, with quota (targeted) sampling not being permitted. In Ireland, the sampling frame is provided by GeoDirectory - an organisation formed by the combined efforts of An Post and Ordinance Survey Ireland. All countries must try to collect a sample of at least 1,500 people, or 800 if the total population of people over 15 is less than two million. The sample size in Ireland fluctuates between 1,800 and about 2,800 depending on the year.

Data collection occurs in face-to-face computer-assisted personal interviews. The response rate target is 70 per cent, and the interviewer must try to contact the interviewee at least four times. However, this response rate is often not reached. Ireland is among the top five countries with regards to response rates, with a 65 per cent rate in 2016 (European Social Survey, 2018).

Similar to the Eurobarometer, the ESS data come with pre-calculated population size weights. There are also two separate weights to be used for both within and across country analysis. A design weight, which is not available in Eurobarometer, adjusts for sampling bias created by the sample design. This ensures that the target sample is representative of the population on a range of variables, including gender, age group, education and geographic region (though there is some variation across countries). The post-stratification weight incorporates the design weight, but makes further adjustment for non-response.

Upon registering an email address, the ESS microdata are free to download from their website. It can be downloaded as an integrated file spanning all countries in the survey or as a country-specific file containing data for one country only. The ESS is a widely used survey, 
with over 100,000 online users and more than 2,700 published works using ESS data existing as of $2017 .^{12}$

At the time of writing, no questionnaire is available for the tenth round of the ESS to be fielded in 2020. However, the ESS have announced that the special modules will be on 'Digital and Social Contacts in Work and Family Life' (which is a new module) and 'Europeans' Understandings and Evaluations of Democracy'. The latter is a repeat of a 2012 module, but we did not identify any relevant attitudinal questions on equality and human rights in the 2012 module so it is unlikely that it will yield any data pertinent to this report.

\subsubsection{International Social Survey Programme}

The ISSP was established in 1984 by Australia, Germany, the United Kingdom and the United States to conduct global surveys on topics related to social science. ${ }^{13}$

The ISSP is conducted every year and is currently run in 42 countries globally. Ireland ceased to be a member in 2013 , but this report examines eight ISSP waves that were run in Ireland between 2000 and 2013.

ISSP surveys are often conducted alongside other national surveys, though they are sometimes run on their own. This means that while an ISSP survey questionnaire is produced every year, the questionnaires are not conducted each year in all countries. Instead, some countries ask multiple ISSP surveys at the same time. The questionnaire topics are selected by a General Meeting of the member nations of the ISSP, which also selects a Drafting Group with members from three to six member nations who write the draft questionnaire. Other member nations can then respond to the initial questionnaire

$12 \mathrm{https}: / /$ www.europeansocialsurvey.org/docs/findings/ESS-Impact-study-Final-report.pdf [last accessed 30 October 2019].

13 All information on the ISSP was sourced from the GESIS and ISSP websites. The links can be found in the References section of this report. 
and it is redrafted. ${ }^{14}$ The documentation does not record the presence of independent questionnaire testing.

In fact, there are considerable inconsistencies in the way that data are collected across countries and over time. No consistent rules regarding sampling method, sample size, data collection method and timing of the ISSP waves are applied. For example, some rounds use a mix of postal and face-to-face surveying, and the 2013 module was conducted between 2012 and 2015 in different countries. The same inconsistencies apply to weighting; for some countries a design weight is provided, for others there is a weight to account for nonresponse and others have no weight at all. This compromises researchers' ability to make comparisons over time and between countries.

In Ireland the ISSP is not typically conducted with other surveys, but usually two years of questionnaires are asked at the same time and often some additional questions are added. The sample size is usually around 1,000, although the 2009 and 2008 waves had sample sizes of just over 2,000. The questionnaires were answered during a face-to-face interview with a paper survey in most years, but a postal survey was used in 2013.

\subsubsection{The European Values Study}

The aim of the European Values Study is to learn about the beliefs, values and opinions of Europeans regarding various aspects of life such as work, religion, family and politics, and to inquire about how moral and social values are changing over time. ${ }^{15}$ The first wave was conducted in 1981 in ten European countries. The questionnaires are developed by the EVS Theory Group and are then reviewed by other working groups within the organisation.

The EVS is conducted every nine years, so since 2000 it has occurred twice, in 2008 and in 2017. This report only examines the 2008 questions, as Ireland did not participate in the

14 http://w.issp.org/fileadmin/user_upload/Rules_and_regulations/ISSP_WP_FINAL_2019.pdf. The ISSP website also contains an online bibliography which details over 9,000 publications which have made use of the data.

15 All information on the EVS was sourced from the European Values Survey website. Further information is in the References section of this report. 
2017 wave. The 2008 wave was conducted in 47 countries in Europe and Eurasia. The EVS website records over 1,600 publications that use the EVS over its various waves. ${ }^{16}$

The EVS uses samples from the population of people of any nationality over 18 years of age in each country. Since 2008, samples have been chosen using multi-stage or stratified probability sampling, though quota sampling was allowed in previous years. The effective sample size was set at 1,500 for the 2008 wave. Data in Ireland were collected in a face-toface interview, sampling about 1,000 people. The response rate in Ireland was 47 per cent in 2008.

Population weights are provided in the 2008 EVS data, but they only match the distribution of the population by age group and gender, meaning that the data may not be representative on a range of other characteristics, such as employment status or family status. Such issues can be addressed by researchers in their analysis of the data.

\subsubsection{The European Union Agency for Fundamental Rights}

The FRA was established as a European Union Agency in 2007 to provide evidence-based advice to policymakers on issues relating to fundamental rights. ${ }^{17}$ The aim of its data collecting work is to inform policy to ensure respect for fundamental rights within Europe.

The FRA conducts surveys on a single human right at a time, rather than covering different issues within one survey. The surveys examined in this report are the European Union Minorities and Discrimination Survey $(2016),{ }^{18}$ the Survey on Violence Against Women in the European Union (2012) and the Survey on the Fundamental Rights of Lesbian, Gay, Bisexual and Transgender People in the European Union (2012). These surveys are carried out in most European Union countries. They are different to the preceding surveys, because

16 https://europeanvaluesstudy.eu/education-dissemination-publications/evs-publications/publications/ [last accessed 30 October 2019].

17 All information on the FRA surveys was sourced from technical reports on the FRA website. The links can be found in the References section of this report. The website does not report on the number of publications using the data.

18 An earlier FRA Survey on Minorities and Discrimination was conducted in 2008. However, this is not included because sampling in Ireland used interviewer-generated and network sampling, and was thus not a probability sampling design (see European Union Agency for Fundamental Rights, 2009). The 2016 survey used a probability sampling design in Ireland and is included in this report. 
they are not surveys of the general population, but instead target certain minority or potentially vulnerable groups. These questionnaires were developed by the FRA with input from consultation events with both stakeholders and academics with expertise on the topic of the survey.

The Survey on Minorities and Discrimination conducted face-to-face interviews with 25,515 respondents of ethnic minority or immigrant backgrounds such as Roma, North African, SubSaharan African and Turkish migrants over the age of 16 across the 28 European Union states. In Ireland, only immigrants from Sub-Saharan Africa or their descendants were interviewed. ${ }^{19}$ The restricted scope of the sample should be noted by any researchers using this data. The response rate was 62 per cent with 425 interviews conducted. The 2011 Census small areas were used as the primary sampling unit in the multi-stage stratified sampling design. Interviews were computer assisted, and weights accounting for design bias and non-response based on selected neighbourhood and individual characteristics are provided. Given the challenges of surveying the minority groups studied, sampling strategies varied somewhat across the countries in the study and users should be cognisant of these differences when using the data and reporting cross-national findings (see European Union Agency for Fundamental Rights, 2017, for more details).

The Violence Against Women Survey was conducted by face-to-face interviews with women aged 18 to 74 years of age. Across the European Union, 42,000 women were interviewed using computer assisted personal interviews. Sampling was done with a two-stage clustered stratified design. The response rate in Ireland was 48 per cent, with 1,567 women interviewed. The average response rate was 42.1 per cent across Europe. Design and poststratification weights are applied to correct for sampling bias and to match the sample to the population on age and rural/urban location. Population size weights are also constructed to allow for cross-national analysis.

19 The selection of the target groups in each country was based on a combination of several considerations. These include: the persons/groups most vulnerable to or at risk of discriminatory treatment following a consultation by FRA with the relevant equality bodies in each Member State; minority groups which maximise the potential to compare the same group in different countries and the size and history of the immigrant and ethnic minority groups in each EU Member State, as well as resources available for the survey (see European Union Agency for Fundamental Rights, 2017). 
The EU LGBT Survey was conducted via an online anonymous questionnaire. It collected data from 93,079 LGBT people aged 18 or over from the EU27 states and Croatia. ${ }^{20}$ In Ireland, 1,625 LGBT people filled in the questionnaire, slightly over half of whom were gay men. As in other surveys, weights are applied to the data. First, weights were used to correct for under- or over-representation of either gay men, lesbian women, people who identify as bisexual and people who identify as transgender. The breakdown by these four categories across the entire sample was calculated from the FRA survey itself, and the data were re-weighted to ensure that this distribution was mirrored in each country. This was to correct for the possibility that some sub-groups (e.g. lesbians) were easier to contact or were more willing to carry out the surveys in some countries than in others. However, it rests on the assumption that each sexual minority group makes up the same share of the population in each country. In addition, population size weights were applied to account for over-sampling of small countries. Despite this complicated and imperfect weighting procedure, the survey's technical report reassuringly notes that applying the weights makes very little difference to the findings of the survey.

The FRA has three forthcoming surveys which could hold information relevant to attitudes towards equality and human rights. One of these is the second FRA survey on the LGBT community, which was fielded in May 2019. According to the FRA, this survey samples intersex respondents for the first time. ${ }^{21}$ Another is the 2018-2019 FRA survey of Roma and Travellers, which takes samples from Belgium, France, Ireland, the Netherlands, Sweden and the UK. Finally, the FRA plans to run a Fundamental Rights Survey which will collect data on people's experiences and opinions concerning the way their fundamental rights are protected..$^{22}$ The technical reports and questionnaires for these surveys are expected to be published during $2020 .^{23}$

\footnotetext{
20 The microdata for the survey are available from the UK data archive. https://beta.ukdataservice.ac.uk/datacatalogue/studies/study?id=7956.

21 The EU Guidelines on Improving the Collection and Use of Equality Data (EU Commission, 2018) stress the importance of consulting rights holders and other relevant experts and stakeholders in devising new categories for inclusion in data collection, and to Incorporate the principles of self-identification, transparency, privacy and accountability in the design.

22 https://fra.europa.eu/en/project/2015/fundamental-rights-survey

23 Personal communication, FRA.
} 


\section{$2.3 \quad$ FREQUENCY OF SURVEYS}

The contribution of each set of surveys to the total number of questions contained in our database is determined by the survey's frequency, length (number of questions) and relevance to attitudes towards equality and human rights. Although the length of surveys is relatively stable, their frequency and relevance vary considerably. For instance, Eurobarometer is a good source simply by virtue of its frequency. It is fielded approximately six times per year. This means that even though there are many rounds which have no relevant questions, the Eurobarometer contributes more than any other survey type to the total question count. By contrast, the FRA surveys are only fielded in 2012 and 2016, but nonetheless serve as a useful source because they are targeted surveys examining discrimination against equality groups.

\section{FIGURE 2.1 TOTAL SELECTED QUESTIONS BY SURVEY, 2000-2018}

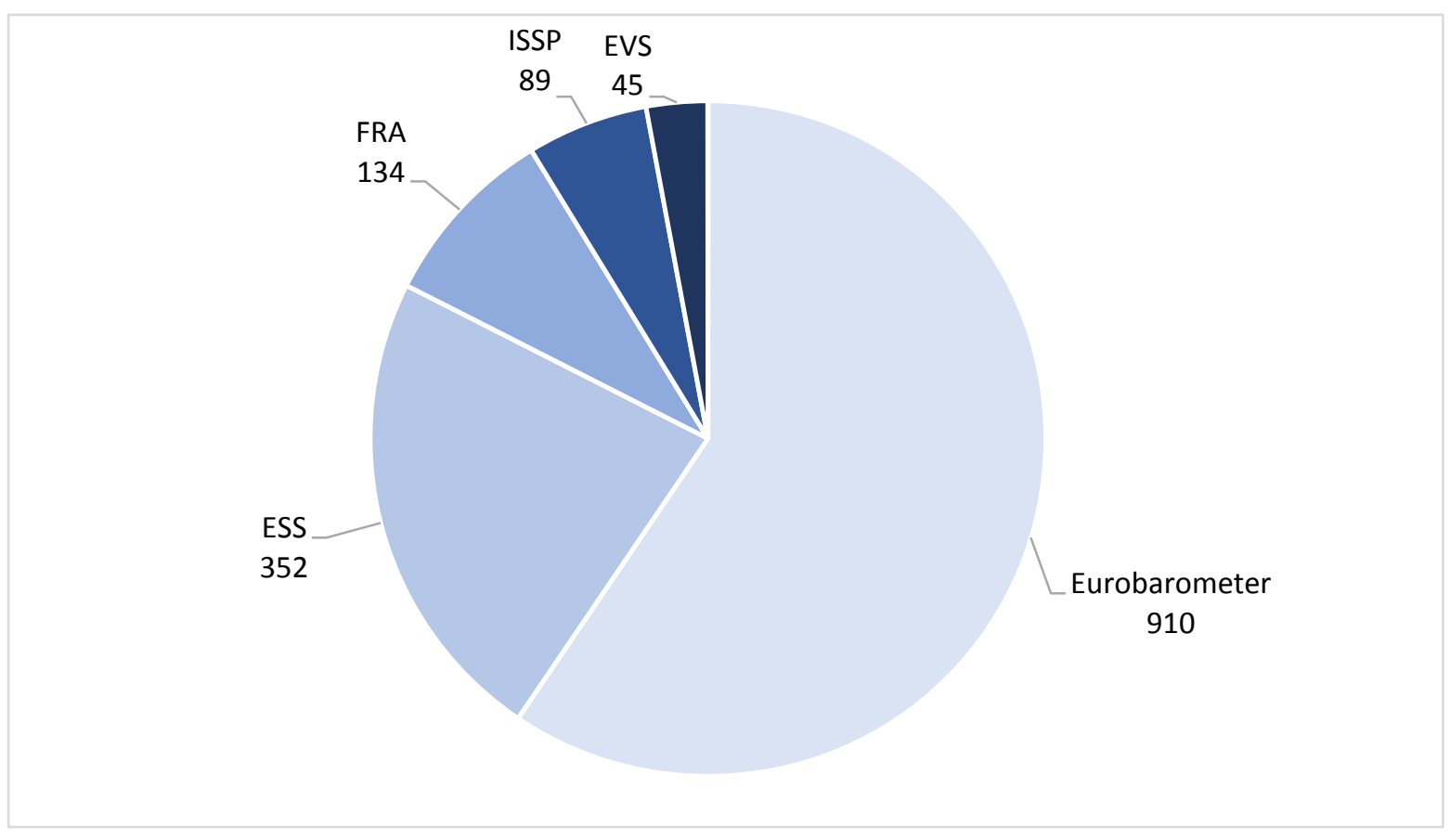

Source: Authors' analysis based on Eurobarometer, ESS, EVS, FRA and ISSP 2000-2018.

Figure 2.1 shows that almost 60 per cent of the questions are fielded in the Eurobarometer survey. In seven of the 19 years considered here, the Eurobarometer is the only survey to have administered questions relevant to equality and human rights (see Figure 2.2). The next largest set of questions (23 per cent) comes from the biennial European Social Survey. The smallest proportion (3 per cent) is attributable to the European Values Study, in which Ireland only participated once over the 2000-2018 period. 


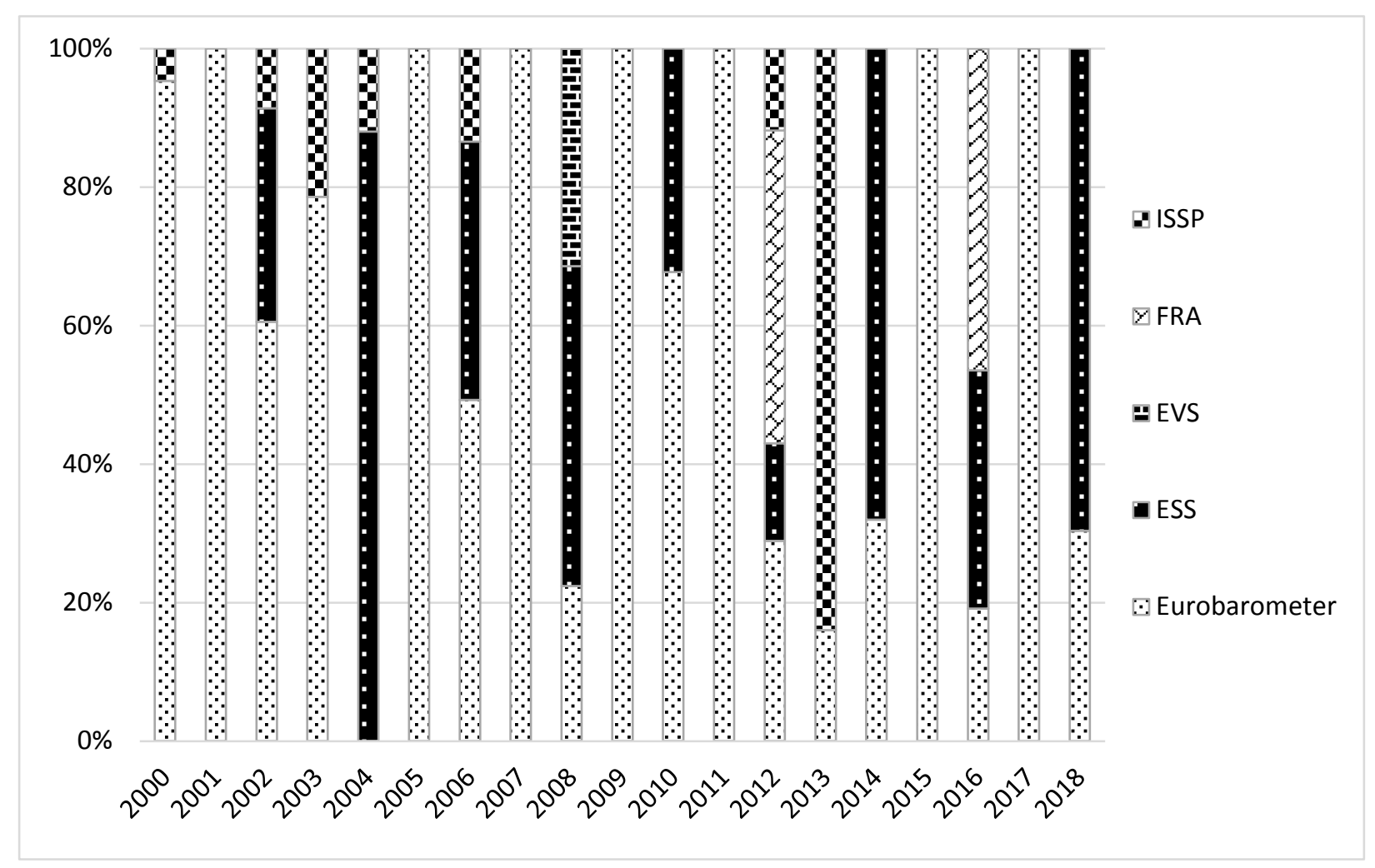

Source: $\quad$ Authors' analysis based on Eurobarometer, ESS, EVS, FRA and ISSP 2000-2018.

Note: The year refers to the year that the survey was fielded rather than the year that the results became available. 


\section{CHAPTER 3}

\section{Equality survey questions}

This chapter provides an overview of the questions asked in the surveys regarding attitudes towards equality. The questions undergo two levels of categorisation. We initially categorise them by the target group of the question. These categories broadly reflect equality groups as set out in Irish legislation:

1. Race, ethnicity, nationality, religion;

2. Gender, civil/family status, and the LGBT community (sexual orientation);

3. Age and disability;

4. Receipt of social welfare.

In this final target group we also consider questions about attitudes to equality in general, such as income, wealth, and social class inequality.

Within these equality groups, we also categorise questions into themes. This is to give the reader an impression of the types of questions that are asked about each equality group. However, given that questions from multiple surveys spanning almost two decades are included, some themes contain a wide-ranging selection of questions. For each target group, we also discuss frequently asked questions which appear five times or more over the 18 years of surveys covered in this report. This study does not cover any questions related to behaviour towards these groups or equality outcomes. Questions about perceived discriminatory experiences are covered in Chapter 4 instead, with the focus in this chapter being on attitudes towards these groups and towards national policies which affect them.

The number of questions on attitudes towards the different groups over the 19 years of surveys varies hugely, from 450 questions on race, ethnicity and nationality to only 31 on religion. There appears to be much more scholarly interest in attitudes towards racial, ethnic and national minorities than other groups. This group comprises around 40 per cent of the total and more than double the number of questions as the next most covered group. Together, the biggest three groups (gender, social welfare and race, ethnicity and nationality) make up almost three-quarters of the total question count. This means that the 
quality and depth of data on attitudes vary enormously between groups, and some groups are under-studied.

\section{FIGURE 3.1 QUESTION COUNT PER TARGET GROUP, ALL SURVEYS 2000-2018}

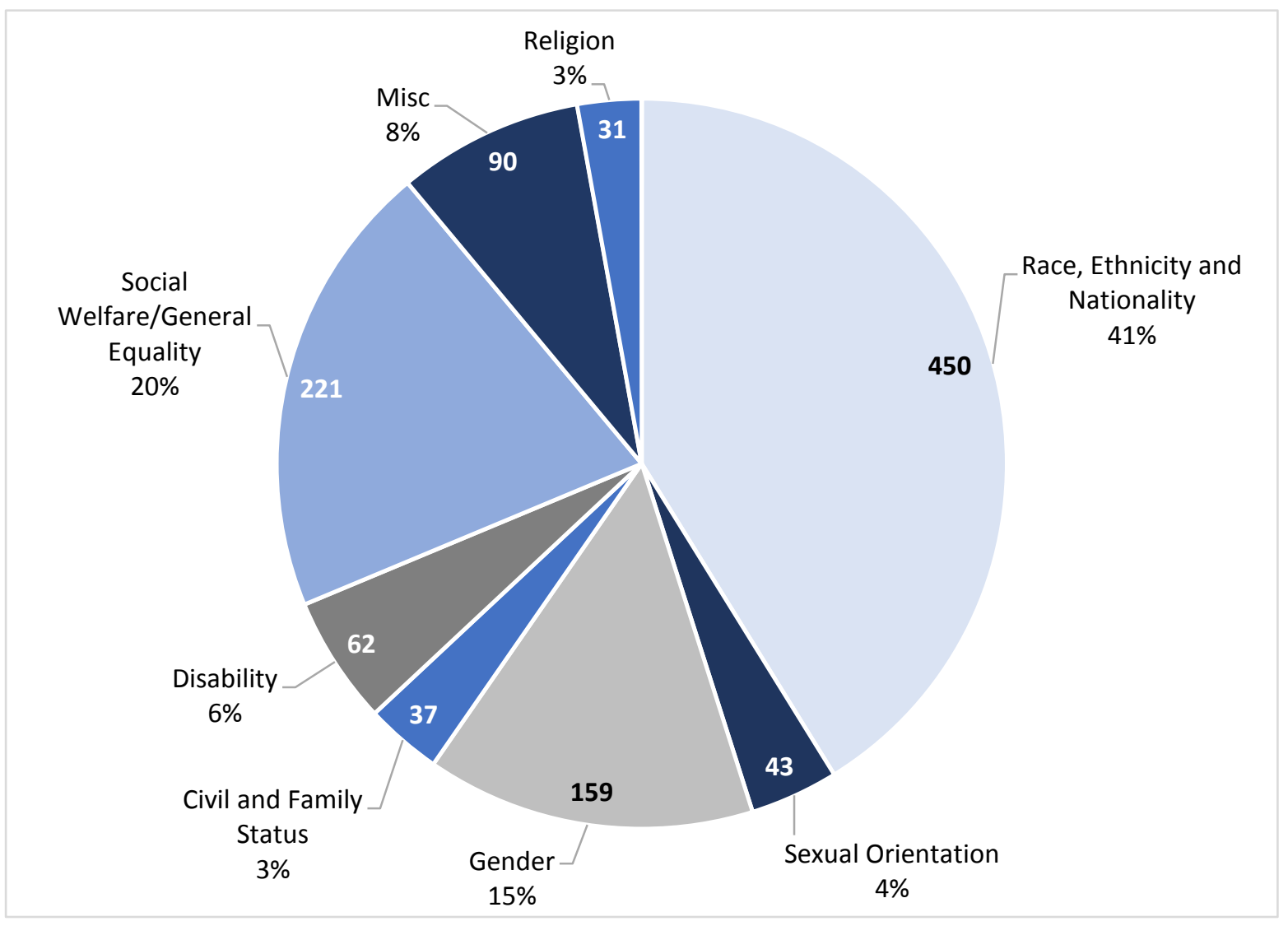

Source: Authors' analysis based on Eurobarometer, ESS, EVS, FRA and ISSP 2000-2018.

\subsection{RACE, ETHNICITY, NATIONALITY AND RELIGION}

\subsubsection{Race, ethnicity and nationality}

Social distance questions are widely used in attitudinal research to indirectly gauge survey respondents' attitudes towards 'out-groups'. Social distance measures the separation of social groups, such as how frequently they interact or how comfortable they are around each other. These questions typically ask how comfortable they are with immigrants or people of other races in various scenarios (e.g. being neighbours, having them as a doctor, being in a relationship with the respondent's child). A slightly less conventional mode of asking about social distance is used in the 2002 ESS. Here respondents are asked to imagine they were trying to find a place to live and are asked if they would choose somewhere that was ethnically homogenous, in a neighbourhood with an ethnic mix, or if it would make no 
difference to them. In this category we also include questions on the related concept of social contact, e.g. how frequently the respondent comes into contact with people of other races or nationalities and whether they are friends with immigrants or racial minorities.

The topic of ethnic minority representation in the media only appears in two years of Eurobarometer and includes inquiries about how much people of ethnic minorities should be covered by national media, and whether the respondent considers immigrants to be portrayed too positively, objectively or not positively enough in the media. The questions on what traits are required to be considered Irish are generally quite similar to each other, asking how important certain characteristics are for a person to be Irish (e.g. whiteness, Catholicism, being born in Ireland, having lived for a long time in Ireland).

Surveys regularly ask respondents what they think about the effect of immigrants on the country. This kind of question appears almost every year. They probe perceptions of the impact of immigrants on various different aspects of Irish life. These can be very specific or very general. The more specific questions ask about the impact of immigration on the economy, culture, crime and the welfare system. The 2000 Eurobarometer module on attitudes to minority groups asks very niche questions on this theme such as whether immigrants improve the country's performance in international sport or whether having children from minority groups in schools can improve the education of all children. Examples of broader questions are whether the respondent agrees or disagrees with the statement 'immigrants contribute a lot to our country' (Eurobarometer, several years) or agree on a scale of one to ten with the statement 'immigrants undermine Ireland's cultural life' (EVS, 2008).

Cultural assimilation questions inquire about attitudes towards immigrant integration into Irish customs. These include topics such as whether immigrants are perceived as wanting to integrate, whether they can or should integrate, and who should be responsible for integration and how (e.g. should the government provide language programmes). One of these questions from the 2013 ISSP asks respondents if immigrants should retain their own culture without adopting Irish culture, should both retain their own culture while also adopting Irish culture, or should forego their own culture in favour of Irish culture. 
Questions relating to views on allowing immigration (legal or illegal) investigate preferences for allowing migrants to enter the country. They often simply ask how much immigration there should be, but also tackle more nuanced issues. For instance, the 2014 ESS special module on immigration asks the respondent for their views on allowing more unskilled labourers and professionals from Poland and Nigeria. These questions are part of a Europe-wide series of questions which ask about attitudes towards workers from poor EU and non-EU countries. Multiple ESS waves also ask who should 'qualify' for immigration to Ireland. These questions allow researchers to discern if there is a hierarchy in public support for different groups of migrants. These include people who are White, wealthy, highly educated, have close family living in Ireland or who speak the official language. As well as attitudes about letting people migrate to Ireland, there are also questions about attitudes to deportation (for instance, whether non-European migrants or migrants with criminal records should be deported).

Less common questions include those on what sort of rights immigrants should have, views on how ethnic minorities are represented in the media, views on asylum seekers and on illegal immigrants, and views on what personal characteristics determine whether a person should be considered Irish. The immigrants' rights category includes questions relating to whether immigrants should have the same rights as citizens. These mainly ask about the broad topic of 'rights', with questions along the lines of 'How much do you agree or disagree that legal immigrants should have the same rights as citizens?' However, there are also some questions on more specific rights such as the 2003 Eurobarometer question on whether immigrants should have the right to vote. This category also includes questions on the respondent's feeling or tolerance towards immigrants or people of other races, whether they think it is right or wrong to treat people of different ethnic origins or nationality differently (e.g. with regards to hiring or refusing housing to them), and whether they approve of anti-discrimination laws.

Questions on asylum seekers typically ask whether Ireland should take in more or fewer asylum seekers. From 2015 onwards Eurobarometer asks the more general question of whether Ireland should help refugees. Multiple waves of the ESS asks whether the Irish government should 'be generous' in granting refugee status. Another type of 
refugee/asylum seeker question asks about why people claim asylum (e.g. that asylum seekers are not in real fear of persecution, asked in the ESS), and about the treatment of asylum seekers (e.g. that they should be allowed to work). The questions regarding illegal immigrants are typically about whether or not they should be deported, as well as questions on whether more needs to be done to tackle illegal immigration, such as punishing those who employ illegal immigrants.

FIGURE 3.2 RACE, ETHNICITY AND NATIONALITY ATTITUDES QUESTION COUNT, ALL SURVEYS

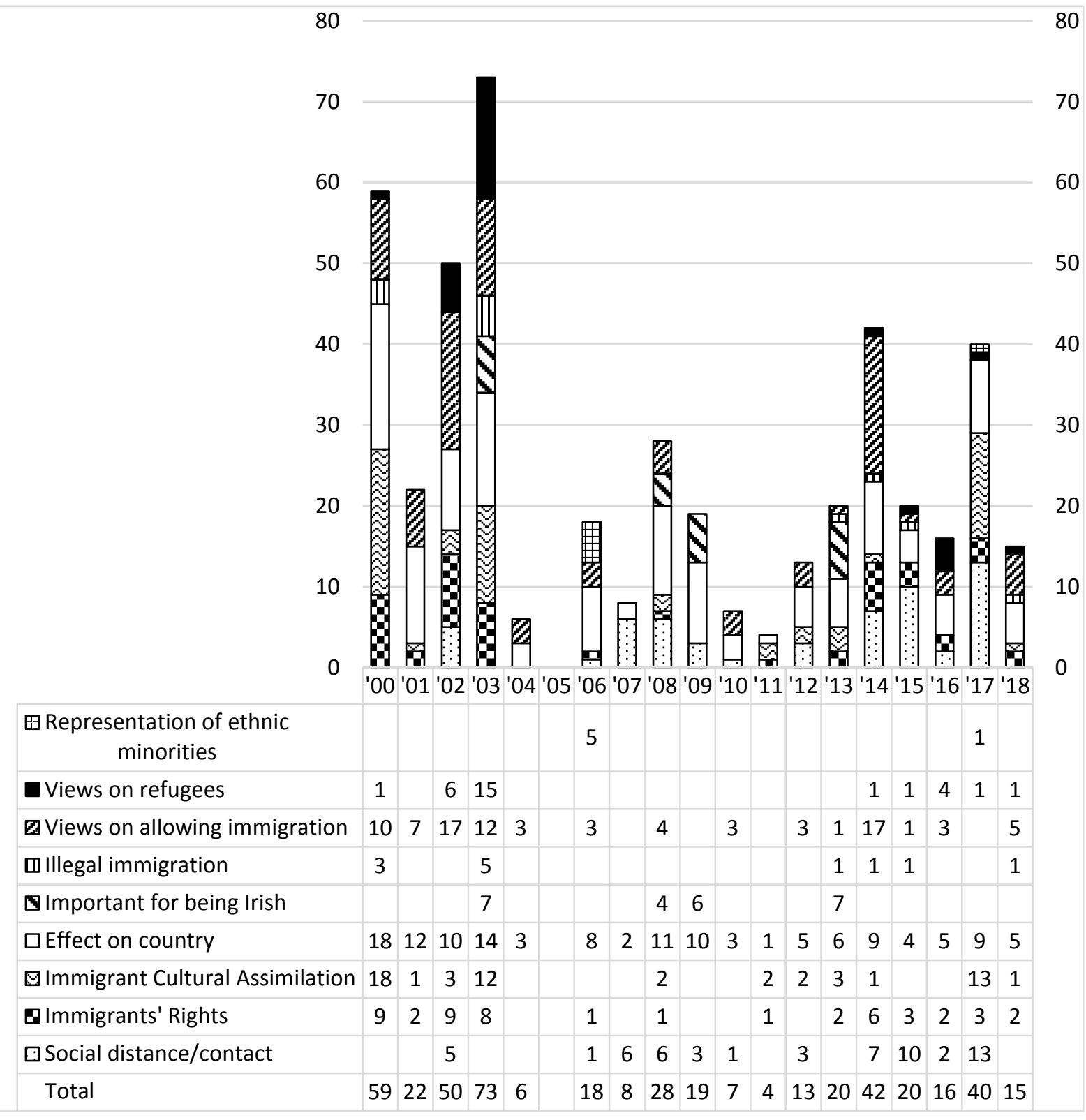

Source: Authors' analysis based on Eurobarometer, ESS, EVS, FRA and ISSP 2000-2018. 
The number of questions on race, ethnicity and nationality peaks in 2000 and 2003 . The large number of questions in 2000 (59) are all fielded in the April-May wave of the Eurobarometer which deals with racism and attitudes to minority groups. The 2003 peak is caused by a Eurobarometer module on 'Issues of Immigration' which contains questions relating to the right to asylum and attitudes towards immigrants and minorities. There is also a 2003 ISSP module on national identity which asks about the personal characteristics that are necessary to be considered Irish.

There are nine questions relating to race, ethnicity and nationality which are repeated five times or more (Table 3.1). Most of these are from the ESS, repeating every wave, and three are from Eurobarometer. The questions that repeat frequently are all about immigration rather than ethnicity. They relate to the effect of immigrants on the country, immigration policy, and tolerance towards migrants. The repetition of the questions across every ESS wave makes it very easy to compare changes in attitudes towards immigrants over time. In the more policy-oriented Eurobarometer survey, respondents were asked about their feelings towards immigration both from inside and outside the EU every year after 2013. 


\begin{tabular}{|c|c|c|c|c|c|c|c|c|c|c|c|c|c|c|c|c|c|c|c|}
\hline & YEAR: & '02 & '03 & '04 & '05 & '06 & '07 & ‘08 & '09 & '10 & '11 & '12 & $' 13$ & '14 & '15 & '16 & '17 & '18 & Total \\
\hline \multirow{6}{*}{ ESS } & $\begin{array}{l}\text { Immigrants make country worse or better place to live 0-10 } \\
\text { scale }\end{array}$ & 1 & & 1 & & 1 & & 1 & & 1 & & 1 & & 1 & & 1 & & 1 & 9 \\
\hline & $\begin{array}{l}\text { To what extent do you think Ireland should allow people of } \\
\text { the same race/ethnic group as most Irish people to come } \\
\text { and live here? } \\
\text { Allow many, some, few, none }\end{array}$ & 1 & & 1 & & 1 & & 1 & & 1 & & 1 & & 1 & & 1 & & 1 & 9 \\
\hline & $\begin{array}{l}\text { To what extent do you think Ireland should allow people } \\
\text { from the poorer countries outside Europe to come and live } \\
\text { here? } \\
\text { Allow many, some, few, none }\end{array}$ & 1 & & 1 & & 1 & & 1 & & 1 & & 1 & & 1 & & 1 & & 1 & 9 \\
\hline & $\begin{array}{l}\text { To what extent do you think Ireland should allow people of } \\
\text { a different race/ethnic group from most Irish people to } \\
\text { come and live here? } \\
\text { Allow many, some, few, none }\end{array}$ & 1 & & 1 & & 1 & & 1 & & 1 & & 1 & & 1 & & 1 & & 1 & 9 \\
\hline & $\begin{array}{l}\text { Country's cultural life undermined or enriched by } \\
\text { immigrants } 0-10 \text { scale }\end{array}$ & 1 & & 1 & & 1 & & 1 & & 1 & & 1 & & 1 & & 1 & & 1 & 9 \\
\hline & $\begin{array}{l}\text { Immigration bad or good for country's economy } \\
0-10 \text { scale }\end{array}$ & 1 & & 1 & & 1 & & 1 & & 1 & & 1 & & 1 & & 1 & & 1 & 8 \\
\hline \multirow{3}{*}{ EB } & $\begin{array}{l}\text { Immigrants contribute a lot to our country } \\
\text { Totally agree, tend to agree, tend to disagree, totally } \\
\text { disagree }\end{array}$ & & 1 & & & & & 1 & & & & 1 & & 1 & 1 & 1 & 1 & 1 & 8 \\
\hline & $\begin{array}{l}\text { Feeling towards immigration: From EU Member States } \\
\text { very positive, fairly positive, fairly negative, very negative }\end{array}$ & & & & & & & & & & & & & 1 & 1 & 1 & 1 & 1 & 5 \\
\hline & $\begin{array}{l}\text { Feeling towards immigration: from outside EU } \\
\text { very positive, fairly positive, fairly negative, very negative }\end{array}$ & & & & & & & & & & & & & 1 & 1 & 1 & 1 & 1 & 5 \\
\hline
\end{tabular}

Source: Authors' analysis based on ESS and Eurobarometer 2000-2018. 


\subsubsection{Religion}

Questions on religion appear much less frequently than those on race or nationality, with most years having no questions at all about religious groups. All questions relate to the social distance/social contact category or a category which we label 'tolerance'. Some questions relating to Muslim immigrants are included in the race, ethnicity and nationality section of this report. Most questions in this section generally relate to immigration in general, rather than any specific group. However, some references are made specifically to Christians, Jews and Muslims. The 2015 Eurobarometer also asks social distance questions about Buddhists and atheists.

\section{FIGURE 3.3 RELIGION ATTITUDES QUESTION COUNT, ALL SURVEYS}

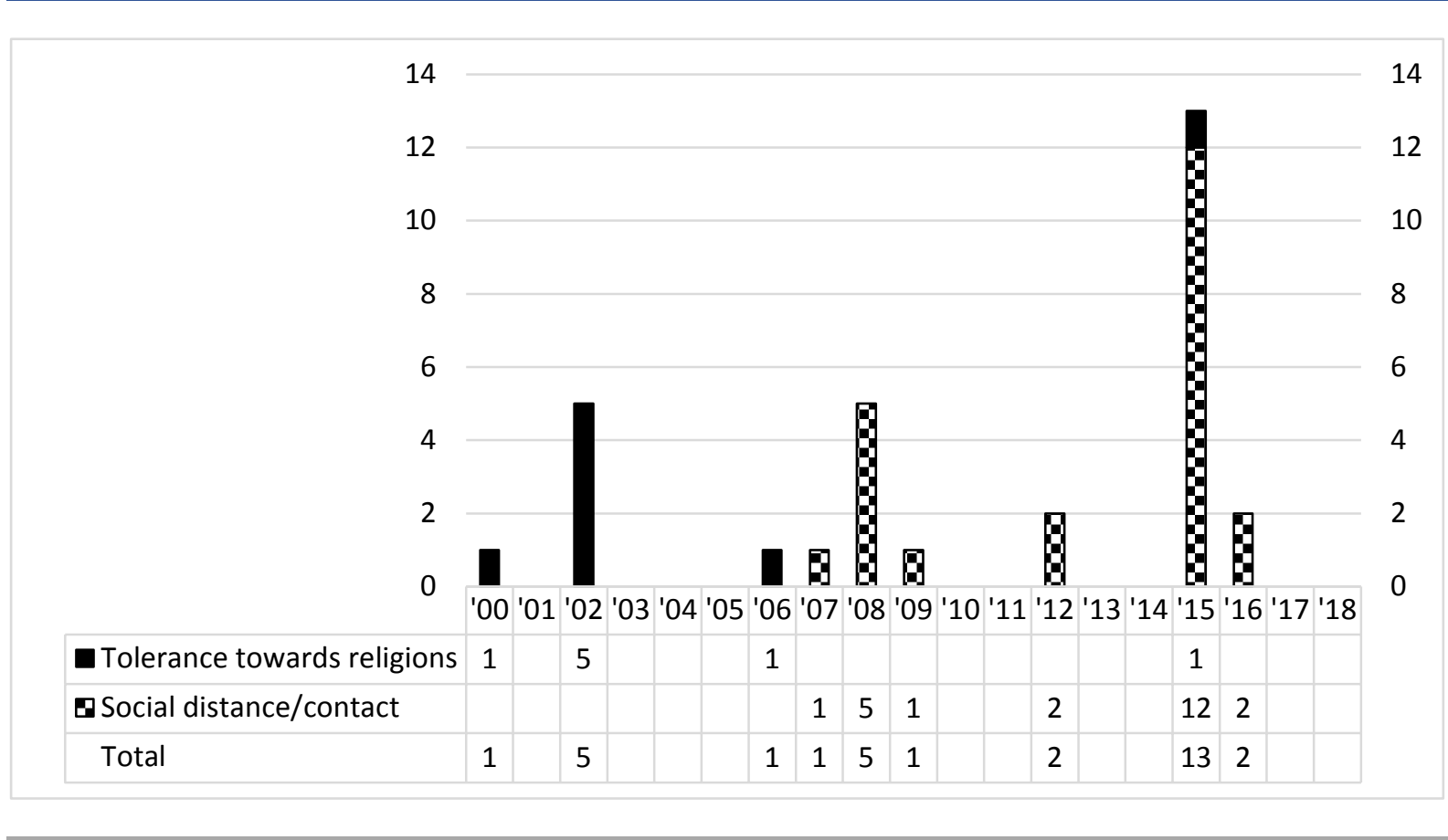

Source: Authors' analysis based on Eurobarometer, ESS, EVS, FRA and ISSP 2000-2018.

Examples of social distance and social contact questions include 'Using a scale from 1-10 how would you feel if one of your colleagues was a Muslim person?' where one signifies being 'not at all comfortable' and ten being 'totally comfortable' (Eurobarometer, 2015) and 'Do you have any friends who have a different religion than yours?' with a yes or no answer (FRA, 2016). 
The tolerance category is broad and captures general feelings towards religious groups. All tolerance questions are fielded in the Eurobarometer, with the exception of a 2002 ESS question which asks respondents if they agree or disagree with the statement that it is better for a country if there is a variety of different religions. A 2002 Eurobarometer question asks participants if they find the presence of people from other religions disturbing. Most other religious tolerance questions ask if it is right or wrong to treat people differently on the basis of religion in areas such as the labour market, housing and private services.

As questions regarding religion are asked much less frequently than race, ethnicity or nationality, there are very few questions on religious groups that repeat frequently. No questions meet the threshold of being asked five times over the course of the period examined.

\subsection{GENDER, CIVIL AND FAMILY STATUS AND SEXUAL ORIENTATION}

\subsubsection{Gender/sex}

Questions concerning respondents' views on gender roles are by far the most commonly asked question on gender. These questions appear almost every year and are more than twice as common as any other gender-related topic. Most measure attitudes relating to the roles of men and women in work and in the home. They include questions on whether mothers or women more generally should work, whether children suffer when mothers or fathers work, whether both men and women should contribute to household income, and whether household and childcare tasks should be shared between husband and wife. There are also questions that inquire about gender stereotypes such as whether respondents agree or disagree with the statement 'it is acceptable for men to cry', or 'women are more likely to make decisions based on emotions' (both from Eurobarometer, 2017).

The questions about views on gender-based violence come entirely from a 2016 Eurobarometer module. These questions try to tap into attitudes about whether genderbased violence is right or wrong and whether it should be illegal. An example of this category is the question 'When is domestic violence against women/men acceptable?', with the options being 'acceptable in all circumstances, acceptable in certain circumstances, unacceptable but should not always be punished by laws, unacceptable and should always 
be punished by law' to try to capture both moral and legal attitudes. It also includes questions on whether intercourse without consent can be appropriate (e.g. if the person was flirting with the assailant). Some of these questions are likely to be affected by framing effects due to how they are phrased, for example a question about whether touching a colleague 'inappropriately' is wrong or not and whether it should be illegal, which is very unlikely to elicit many responses saying it is not wrong, due to the behaviour being described as 'inappropriate' in the question. There is also a high risk of socially desirable responding associated with these questions.

The views on gender equality theme includes questions on whether men and women should be treated equally or considered as equal generally. For example, a 2014 Eurobarometer question asks respondents if they believe that equality between men and women is a fundamental right. ${ }^{24}$ Questions relating to employment are also common. A standard question in this domain asks whether men should have more right to a job than women if work is scarce. Questions relating to perceived obstacles to women being in power tap into views about why women are less frequently in positions of responsibility, (e.g. that women are less interested in them or less qualified for them), as well as perceptions of whether there are obstacles facing women, for example within companies or within political parties. Many of these questions are concentrated in the 2011 Eurobarometer module on 'Women in decision-making positions'. The smallest category relating to gender is about social distance, which is mostly about how comfortable participants would be on a scale of one to ten having women as elected politicians (asked in several years of Eurobarometer), and if the respondent thinks there are enough women in power in politics or business.

The year with the largest number of questions on gender is 2016 , which is due to the Eurobarometer module on gender-based violence. There was a slightly larger number of questions in 2017, which are from a Eurobarometer module on gender equality. All the surveys examined in this report included questions on gender attitudes.

24 We note that the questions refer to men/women rather than male or female but that it is not clear if the intention was to capture gender or sex and if such a distinction was made by respondents. 


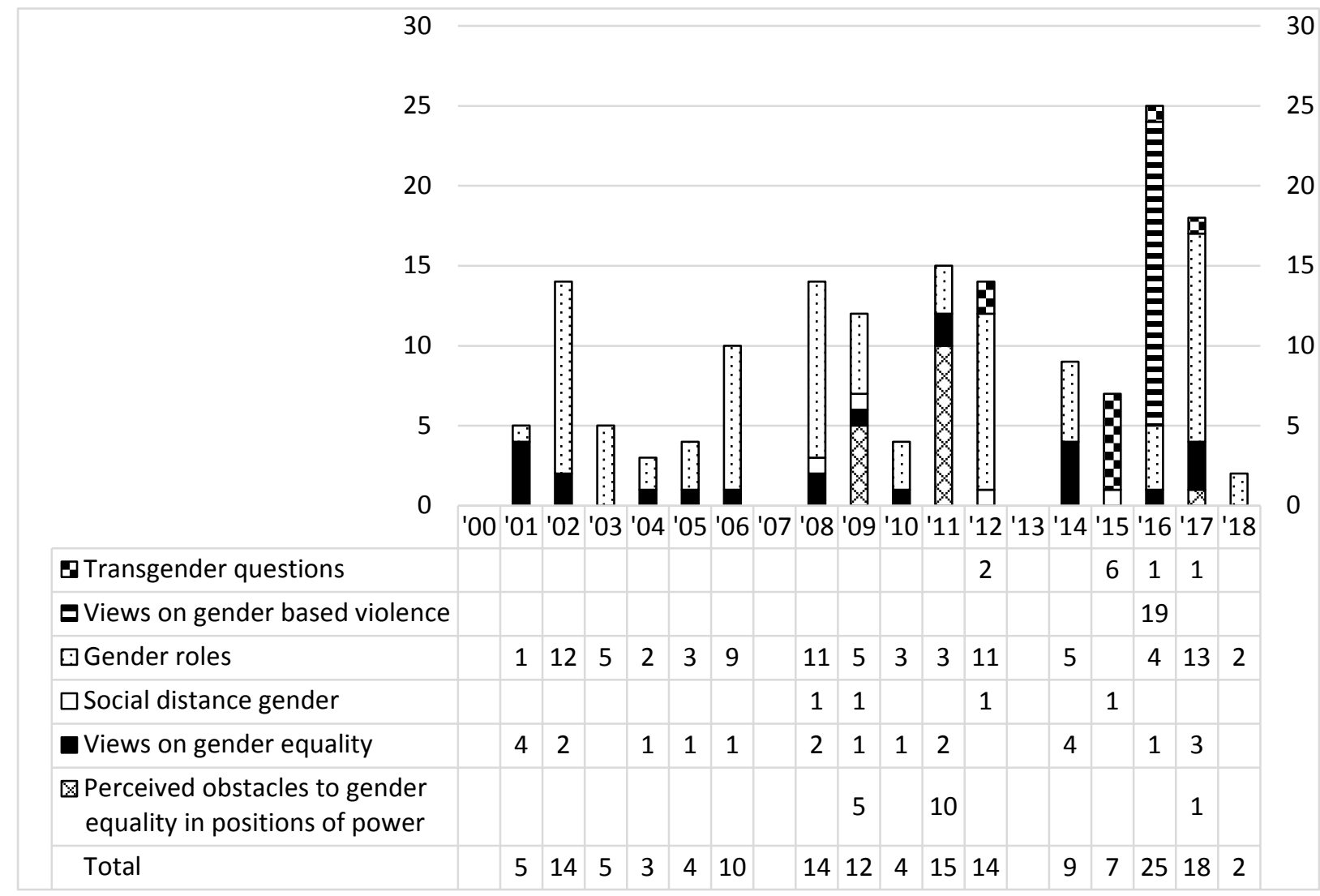

Source: Authors' analysis based on Eurobarometer, ESS, EVS, FRA and ISSP 2000-2018.

No questions relating to gender meet the threshold of being asked five times or more in the same format between 2000 and 2018, despite the relatively large number of total questions on gender.

There is also a small number of questions that ask about attitudes to transgender people, which only begin to be asked in 2012. Because of the small number of questions, all the transgender questions were grouped together, so this category includes questions on social distance from transgender people (the majority of the questions), a question about whether children should be taught about diversity of gender identities, and a question about whether transgender people should be able to change their gender on their civil documents. These questions appear in the Eurobarometer in modules on discrimination in 2012 and 2015 and on fairness and inequality in 2017. 


\subsubsection{Civil and family status}

Civil and family status are the least frequently asked about target groups, which is why they are grouped together in this report. There are only 25 questions relating to these groups over all the surveys and several years contain no questions about them. Almost half of the questions relate to views on unmarried families, which include attitudes towards couples living together, having sexual intercourse or having children before marriage. Examples of these questions include agreeing or disagreeing with the statement 'People who want children ought to get married' (ISSP, 2002/2012) and 'Do you think it is wrong or not wrong if a man and a woman have sexual relations before marriage?' (Eurobarometer, 2001).

Questions regarding views on family policy are about whether the government should be responsible for providing childcare services or paid leave to working parents for looking after sick family members. These are all from the 2008 and 2016 waves of the ESS.

Tolerance towards family and civil status is measured by questions asking about approval or disapproval of certain family situations such as divorce or childlessness. For instance, the 2008 EVS asks if the respondent 'justifies divorce'. This question is asked as part of a battery of items on social attitudes which also consider other moral issues such as abortion, adultery and euthanasia. Questions about attitudes towards lone parents are mostly about whether one parent can raise a child as well as two parents can, and one question from the 2008 EVS asks whether the respondent approves or disapproves of single mothers.

The increase in questions on family and civil status in 2008 is due to the EVS, and in 2006 is due to an ESS module on the family. The questions about civil and family status are mainly from the ESS and ISSP, with only one from Eurobarometer, making it by far the least covered group by Eurobarometer.

Given the very small number of questions corresponding to this equality group, unsurprisingly there are no questions that appear five times or more. 


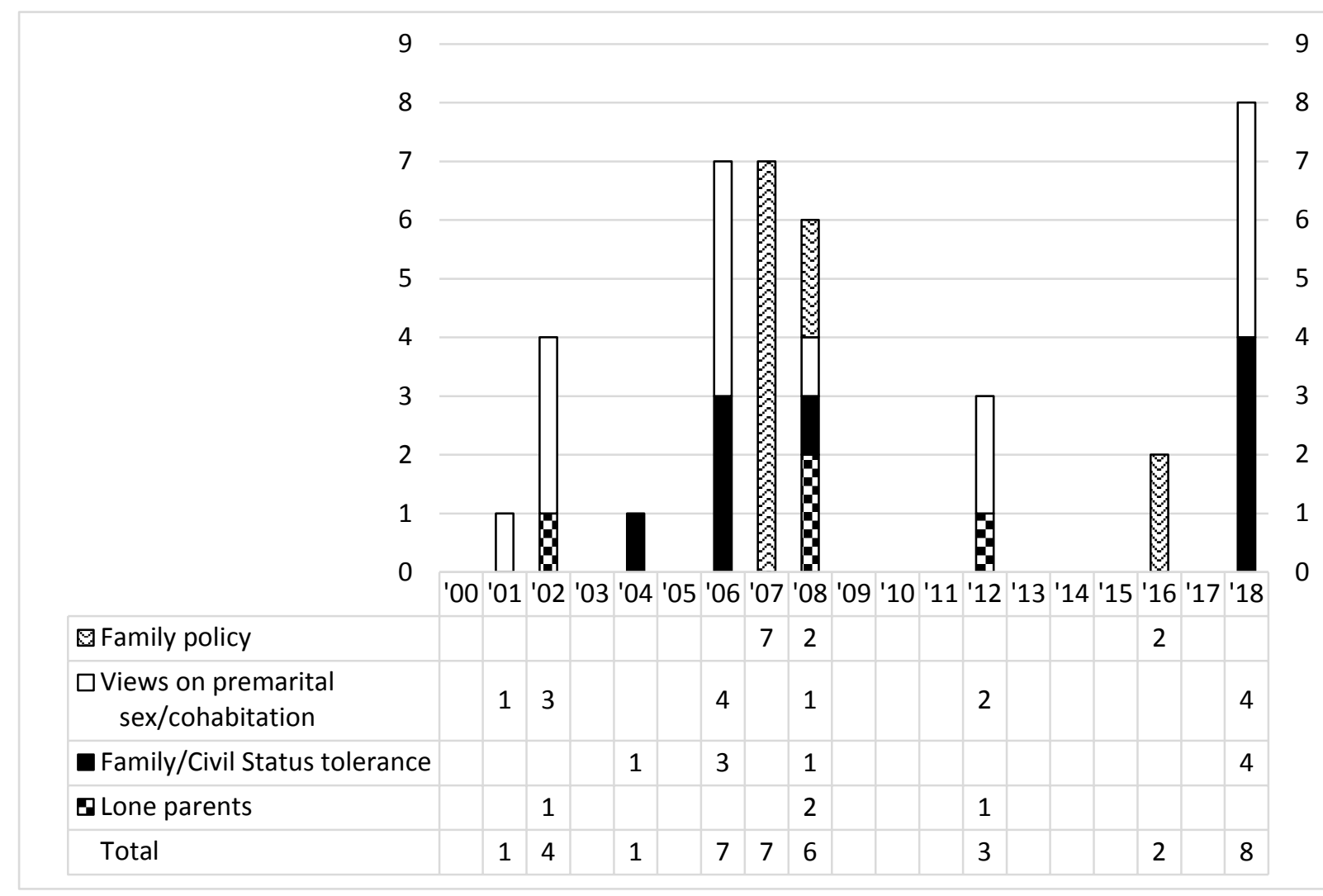

Source: Authors' analysis based on ESS, Eurobarometer, EVS and ISSP 2000-2018.

\subsubsection{Sexual orientation}

Questions relating to sexual orientation are asked in most years, although many survey waves have only a handful of questions on the topic. The most common questions are those about tolerance of non-heterosexual people, which comprises half of all the questions about this group. This category inquires mainly about attitudes towards equal treatment of lesbian, gay or bisexual people and towards displays of affection by same-sex couples. Examples of these include 'How comfortable would you feel with gay couples showing affection in public? (e.g. kissing or holding hands)' (Eurobarometer, 2015) and 'Is it wrong to refuse a promotion on the basis of an applicant being homosexual?' (Eurobarometer, 2002). As part of the same series of questions which asks if the respondent justifies divorce, the 2008 EVS asks respondents to rate on a scale of 0-10 to what extent they think homosexuality 'can be justified'.

The social distance/social contact category is similar to social distance questions in relation to other equality groups, asking questions such as whether respondents would be 
comfortable with a homosexual person being an elected politician or a neighbour, as well as asking whether the respondent has any lesbian, gay or bisexual friends (asked in several waves of Eurobarometer). The smallest number of questions relating to sexual orientation are asked about views on same sex parenthood and marriage. This category examines attitudes about whether same sex couples should be allowed to adopt or whether they can raise children as well as heterosexual couples. Some surveys, including a 2001 Eurobarometer module targeted at young people (aged 15-24), also asked about whether same sex couples should be allowed to be married.

The year with the highest number of questions on sexual orientation is 2015 , due to the Eurobarometer module on discrimination. Eurobarometer asks the most questions on sexual orientation of all the surveys. Regarding terminology, most of the surveys use the terms 'sexual orientation', 'homosexual', or 'gays and lesbians' in their questions. The use of the term 'bisexual' in questions only begins in 2012.

Only one question on sexual orientation appears more than five times over the surveys, appearing in eight different ESS waves. This question requires respondents to say whether or not they agree with the statement 'Gays and lesbians should be free to live life as they wish', with 'Agree strongly', 'agree', 'neither agree nor disagree', 'disagree', or 'disagree strongly' as the potential response options. This question does not change between waves, making it easy to track potential changes in attitudes over time. 
12

10

8

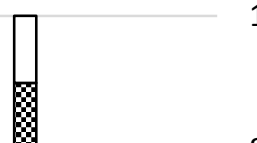

6

4

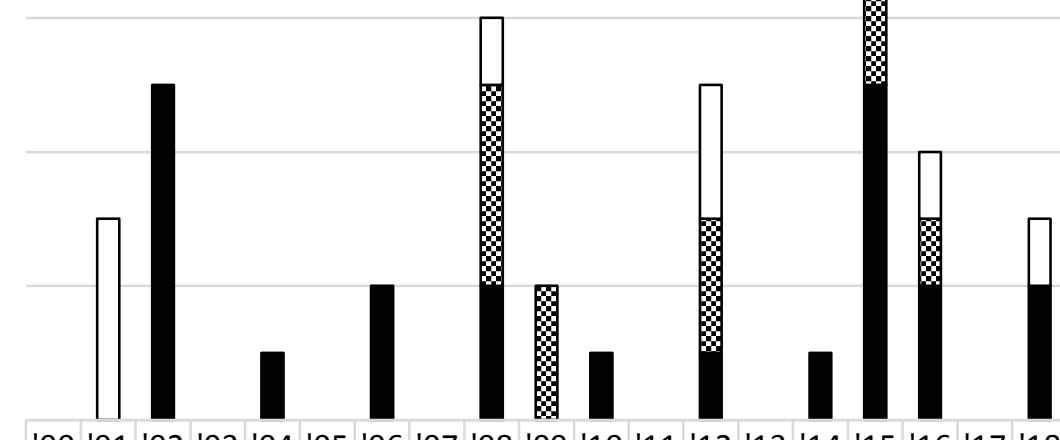

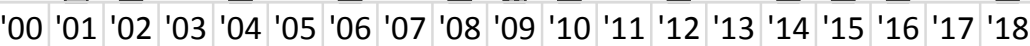

$\square$ Views on LGBT parents and marriage

Social distance/contact

- Tolerance

Total
3

1

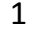

32

2

$6 \quad 2 \quad 1$

\begin{tabular}{|c|c|c|c|c|c|}
\hline 2 & & 1 & 1 & & 1 \\
\hline 2 & & 4 & 1 & \\
\hline 1 & 1 & 5 & 2 & 2 \\
\hline 5 & 1 & 10 & 4 & 3 \\
\hline
\end{tabular}

Source: Authors' analysis based on Eurobarometer, ESS, EVS, FRA and ISSP 2000-2018.

\subsection{AGE AND DISABILITY}

\subsubsection{Age}

Attitudinal questions relating to age are quite common in the surveys, being asked more frequently than religion and sexual orientation, and at a similar frequency to disability. The social distance/social contact questions about this group are similar to those about other groups and relate to several different age groups such as over 55 , over 75 , under 30 and under 25. The most common question in this theme is to ask how comfortable on a scale of one to ten the respondent is with an elected politician being either over 75 or under 30 . The Eurobarometer also asks how comfortable the respondent would be with having a colleague at work who is aged over 60 , and another question probes the equivalent attitude regarding colleagues under the age of 25 . The social distance questions are all from Eurobarometer modules on discrimination, but there are two social contact questions from the 
2008 ESS module on ageism which ask how many friends the participant has who are aged under 30 and over 70.

Questions on attitudes towards older and younger people are varied and are difficult to categorise into meaningful themes. We consider views on older people, on young people and on equality between the ages separately. Questions concerning views about older and young people are quite similar, relating to those over 70 and those in their 20 s. These inquire about things such as how positively the respondent feels about those age groups and how much they think they contribute to the economy. There are also some questions specific to the age groups. For instance, included in the 'views on older people' theme is a question from the 2008 ESS module on ageism on how much of a burden people over 70 are on the health service. That same module also asks how worried the respondent is about crime committed by people in their 20s. The theme 'Views on equality between ages' includes questions on whether it is right or wrong to deny an applicant a job due to them being under 25 years old, whether young patients should be prioritised over older ones (both of which are fielded in the 2002 Eurobarometer), opinions of employment equality legislation based on age and how age-friendly the respondent views their country to be.

Questions on tolerance to older workers are relatively common so we make them a separate theme, distinct from 'views on older people'. This theme mainly includes questions from a 2011 Eurobarometer wave on stereotypes of workers over 55, such as whether they are more likely than younger workers to be 'experienced' or 'up to date'. It also includes questions relating to whether it is right or wrong to refuse a job to someone aged older than 55 , and whether older workers should retire to make way for younger people in the labour market (Eurobarometer, 2001). The theme of views on government responsibility for older people mainly consists of questions about whether the government should guarantee older people a certain standard of living and if it should spend more, the same or less money on pensions.

The highest number of questions relating to age appears in 2011, mainly due to a Eurobarometer module on 'Active aging' which includes multiple questions on older workers. Numerous questions were also asked in 2008, mainly due to the ESS wave that year. Most of the questions relating to age are from Eurobarometer, although the ESS also 
has quite a few. There are no attitudinal questions about age in the ISSP, EVS or FRA. There is a shortage of data available on attitudes towards age groups in recent years. After 2011, only seven age-related questions are asked overall. There were no questions relating to age that appeared five times or more.

\section{FIGURE 3.7 AGE ATTITUDES QUESTION COUNT, ALL SURVEYS}

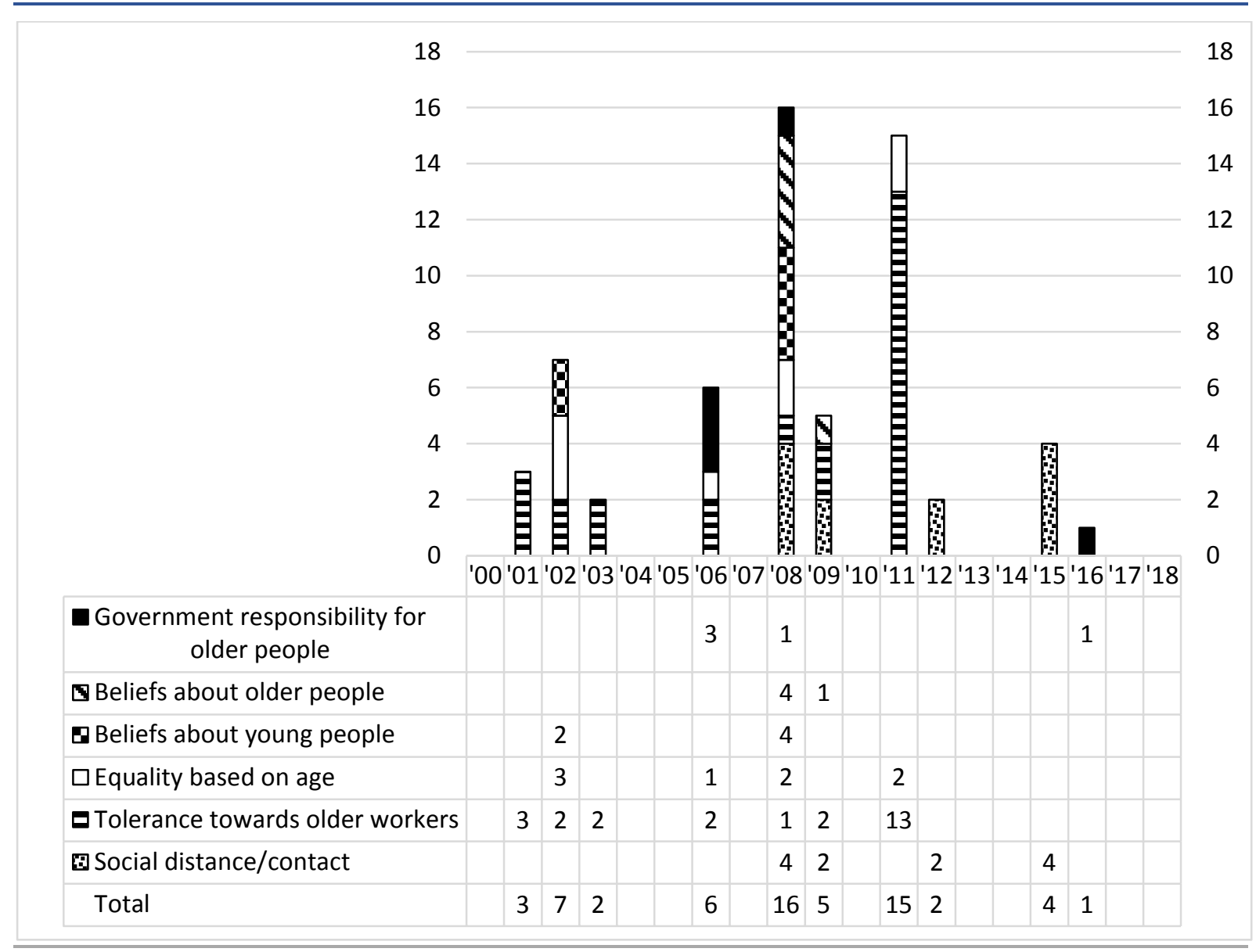

Source: Authors' analysis based on ESS and Eurobarometer 2000-2018.

\subsubsection{Disability}

Questions on attitudes relating to disability are asked in most years of the surveys, but about half of the questions on disability were asked in 2001 and 2002, with very low numbers of questions asked each year after 2003. This means that there is a likely shortage of up-to-date comparative data on attitudes towards people with disabilities. In most questions, participants are asked simply about 'disabled people' or 'people with disability', without asking about a specific disability. However, some Eurobarometer questions differentiate between 'physical disability' and 'mental illness', although only one question overall references intellectual disabilities. The most frequent topic is social distance, which 
asks similar questions to all the other social distance topics. For instance, the 2001 Eurobarometer module Europeans and Disability asks 'Do you think you would feel at ease, or not at ease, with a person in a wheelchair working as a GP?'. This is the only module that specifically asks about people in wheelchairs.

Questions relating to tolerance are asked less frequently and are concentrated in 2002 (Eurobarometer). These questions are to do with respondents' general views about people with disabilities. For instance, one question asks whether the respondent believes that disabled people are less productive in the workplace (Eurobarometer, 2001). There are also several questions about whether it is right or wrong to treat disabled people differently, such as refusing a promotion due to their disability. No tolerance questions are asked after 2010.

The theme of government disability policies relates to questions about how people with a disability should be treated, for example, asking whether children with a disability should be in the same schools as children without a disability, or whether more money should be spent to remove barriers which complicate the lives of people with a physical disability (Eurobarometer, 2001). These stop being asked after 2006 except for one Eurobarometer question in 2012 which asks if the respondent believes that not providing sufficient accessibility constitutes discrimination.

The large number of questions on disability in 2001 is due to a Eurobarometer module on people with a disability. There was also a Eurobarometer module on disability in 2003 and on discrimination in 2002, which increased the question counts for those years. There are very few questions on disability from surveys other than Eurobarometer, and none at all in the ISSP or EVS. There were no questions on disability that occurred five times or more. 


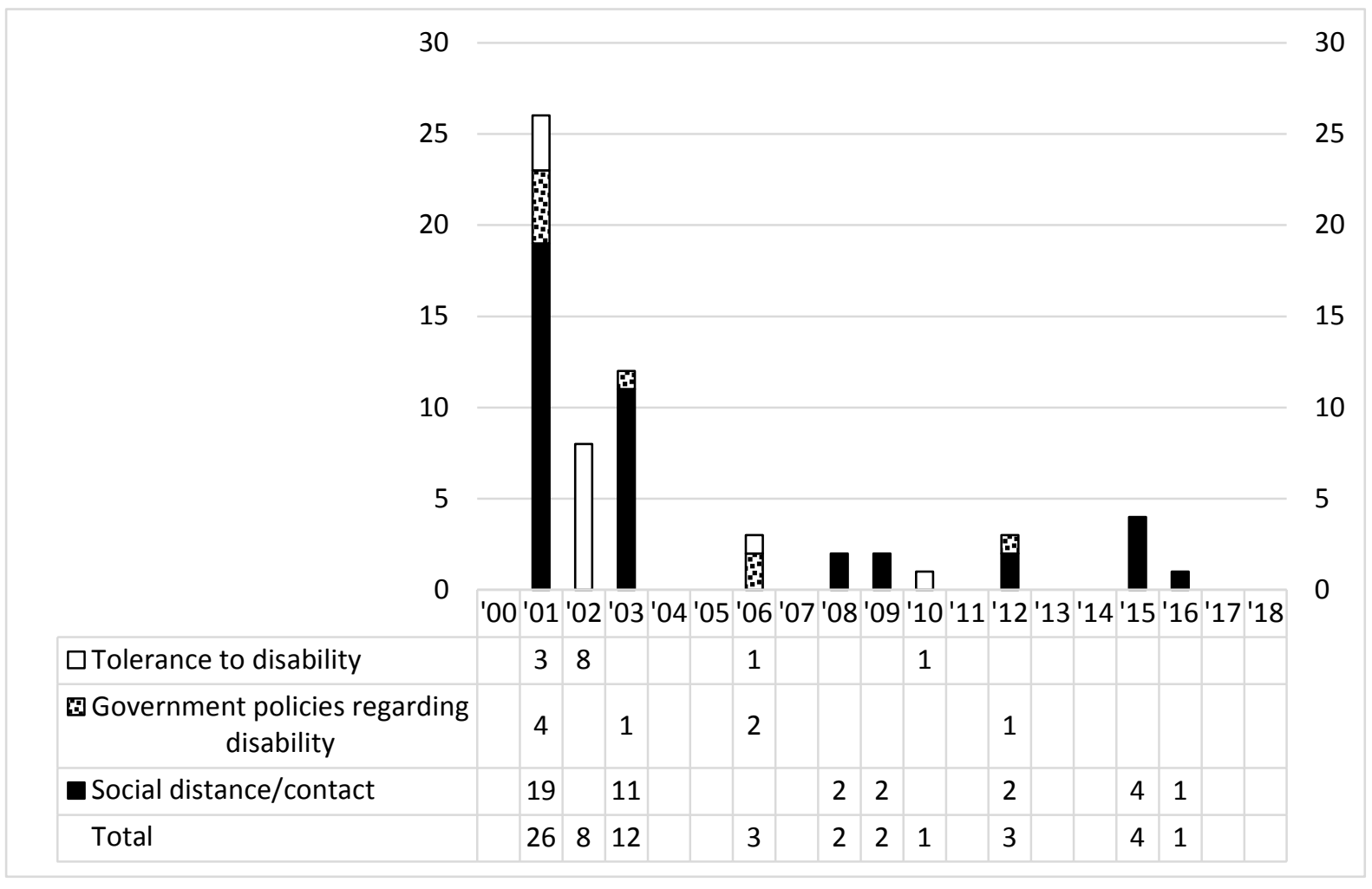

Source: Authors' analysis based on Eurobarometer, FRA and ESS 2000-2018.

\subsection{ATTITUDES TOWARDS SOCIAL WELFARE AND EQUALITY IN GENERAL}

\subsubsection{Social welfare}

Questions regarding attitudes towards social welfare and poor people appear very frequently, with slightly more questions being asked about this than about gender, another large category. These questions provide an insight into public perceptions of socio-economic rights. About half of them relate to attitudes about why people are poor or homeless, which are all from the Eurobarometer, except for one question from the EVS. This theme includes questions such as 'why are people homeless?' where the respondent can choose several responses from a set of options, including factors that are external to the person such as 'they have no access to support' as well as factors the person can control such as 'they choose to live this way'. The questions about poverty are phrased in a similar way. Only one question from this theme is asked after 2010, but variations of these questions are asked in Eurobarometer in 2001, 2007, 2008, 2009 and 2010 modules. The theme of views on social welfare includes questions about what effects the respondent thinks social welfare has (e.g. costs too much, makes people lazy, prevents poverty), as well as multiple questions about whether unemployed people on social welfare should have to take any jobs 
they are offered in various scenarios, such as if they are single parents or if the job requires less education than they have. Three of these questions are fielded in the EVS but the rest come from the 2008 and 2016 waves of the ESS on 'welfare attitudes'.

Views on equality of income are measured by questions about whether the respondent considers income differences to be a problem, such as asking if respondents consider income differences in their country to be too large in Eurobarometer in 2001, or whether large differences in income are acceptable to reward talents or effort in the 2008 and 2016 ESS. Questions about the government's responsibility for poverty inquire about what the respondent thinks the government should do about poverty. This includes topics such as whether the government should provide people with housing, healthcare or jobs. A potential weakness of some of these questions is that they do not mention that such spending programmes may entail tax increases. However, other questions probe whether the government should be responsible for the redistribution of wealth, or ask whether the rich should pay more in tax to fund anti-poverty measures.

Social welfare questions are asked most years, with usually either very few questions or a very large number of questions related to it being asked. The years with the highest number of questions are 2009 and 2010. This is mainly due to a large number of questions on why people are poor or homeless being asked in Eurobarometer modules on poverty and social exclusion. There are also peaks in 2008 and 2016 from ESS modules on welfare attitudes. All surveys except the FRA include questions on social welfare.

Only one question on social welfare appears more than five times over the course of the surveys, appearing in eight waves of the ESS. This question requires respondents to respond that they 'Agree strongly', 'agree', 'neither agree nor disagree', 'disagree' or 'disagree strongly' with the statement 'the government should take measures to reduce differences in income levels'. This is included in the government responsibility for poverty category. 


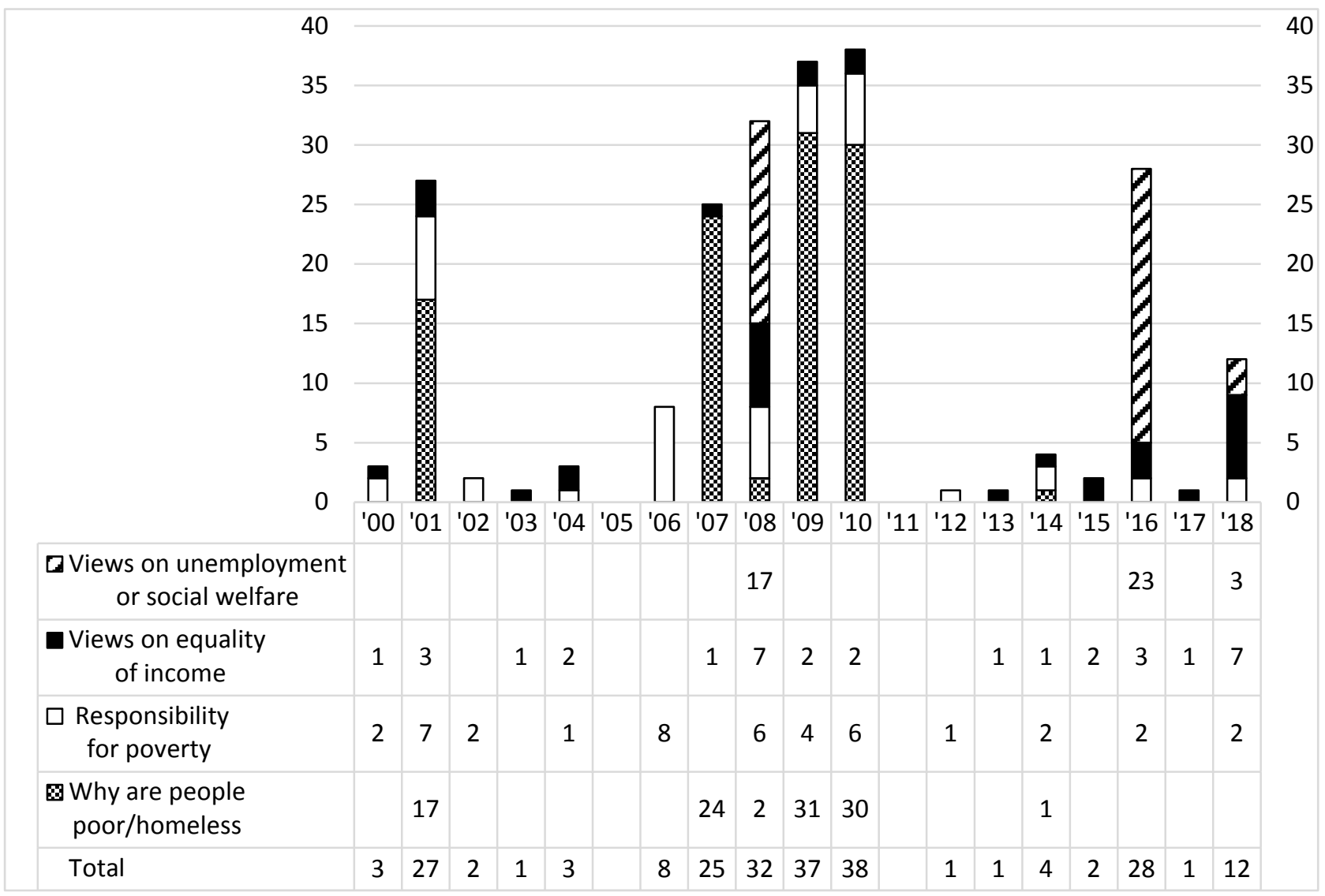

Source: Authors' analysis based on Eurobarometer, ESS, EVS and ISSP 2000-2018.

\subsubsection{Miscellaneous equality and human rights attitude questions}

The miscellaneous category includes attitudes towards equality or human rights that do not relate to any of the target groups mentioned above or to social welfare. The bulk of these questions are about important personal values of the respondent which are relevant to equality and human rights in general. In this type of question, the respondent is often asked to choose from a list of options (such as tolerance, human rights or equality) which values are among the most important for them personally. Also included in this category are ESS questions in which the respondent is given a description of a hypothetical person, and is asked how similar they are to this imaginary character. For instance, participants are asked if they consider themselves to be like a person who 'believes everyone should have equal opportunities in life'.

Other questions on values relate to post-materialist values (Inglehart, 1977) - the extent to which the respondent subscribes to a value orientation that emphasises self-expression and 
quality of life over economic resources and physical security. Post-materialist values include a greater emphasis on human rights. Post-materialist values are captured through a standard four-item index, which asks about traditional values and post-materialist values. These questions are asked twice in 2008, once in the Eurobarometer and once in the EVS. The question in the EVS asks 'There is a lot of talk these days about what the aims of this country should be for the next ten years. On this card are listed some of the goals which different people would give top priority. If you had to choose, which of the things on this card would you say is most important?' with the options on the card being 'maintaining order in the nation', 'fighting rising prices', 'giving people more say in important government decisions' and 'protecting freedom of speech'. The first two items are considered traditional values and the latter two items post-materialist values.

There are few questions that refer directly to the protection of human rights, with five questions overall. ${ }^{25}$ This category includes several different topics such as whether it is important that the government protects minority rights, whether freedom should be traded off for equality, and whether it is important to live in a country with human rights or equality of opportunity. The human rights questions included in this analysis are all asked either in the ISSP or the Eurobarometer.

The anti-discrimination action category consists of questions from Eurobarometer 2017 about how the respondent would act if they discovered there was a gender pay gap at work, with options such as quitting their job or doing nothing. These are included in this chapter rather than Chapter 4 as they are hypothetical questions about respondents' anticipated actions in the face of discrimination, while Chapter 4 deals with questions about discrimination that has actually happened. The questions on miscellaneous equality policies only occur in 2000 and 2001, and address questions about how the government should integrate 'out-groups' and help minorities in general. They are from Eurobarometer modules on attitudes towards minorities and immigrants, and attitudes towards out-groups.

25 Questions on discrimination and unfavourable attitudes towards different groups protected under human rights law mean that many of the questions discussed in previous sections relating to specific groups are relevant to human rights. Here we include questions that directly assess respondents' attitudes to the protection of human rights. 
The year with the most frequent number of questions is 2017 , because of the Eurobarometer questions on anti-discrimination action, which were in a module on gender equality.

FIGURE 3.10 MISCELLANEOUS EQUALITY AND HUMAN RIGHTS QUESTION COUNT, ALL SURVEYS

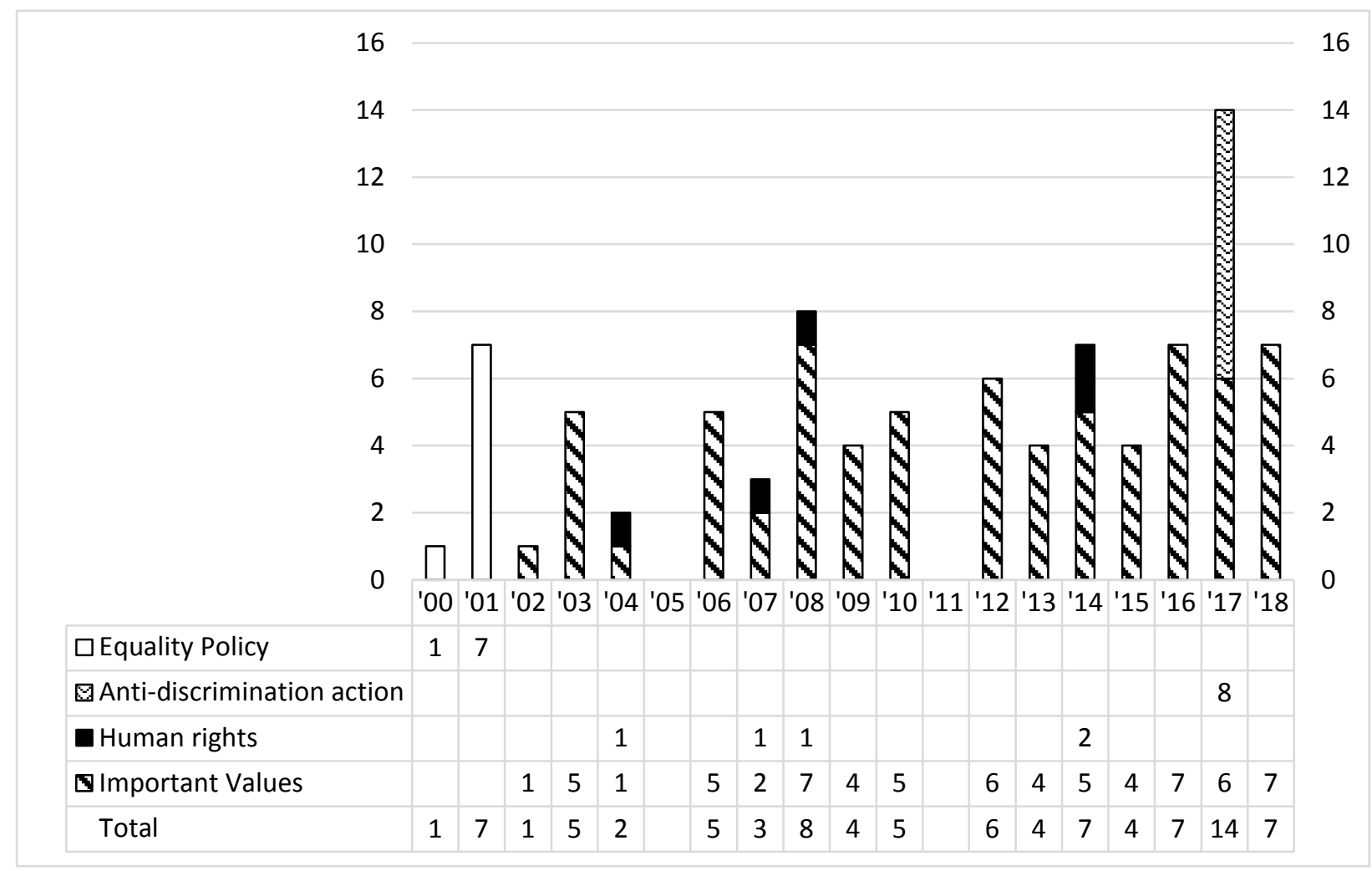

Source: Authors' analysis based on Eurobarometer, ESS, EVS, FRA and ISSP 2000-2018.

There are three questions which repeat five times or more in the miscellaneous category. The question 'In the following list, which are the most important values for you personally?' appears in 12 Eurobarometer survey years, the most of any question in the surveys (Table 3.2). The respondent can choose three of 12 potential options. These options are; rule of law, respect for human life, human rights, individual freedom, democracy, peace, equality, solidarity, tolerance, religion, self-fulfilment and respect for cultures. The next most common questions ask the respondent if they consider themselves to be like a person described in the survey. 'She/he thinks it is important that every person in the world should be treated equally. She/he believes everyone should have equal opportunities in life.' This appears in eight ESS waves. The respondent can select the options 'Very much like me', 'like me', 'somewhat like me', 'a little like me', 'not like me', or 'not like me at all'. 


\subsection{INTERSECTIONALITY}

Intersectionality refers to the way in which the combination of equality characteristics, e.g. gender and ethnicity, may lead to differential treatment or outcomes. We found few questions in the surveys analysed that assess whether attitudes vary when the subject of the attitudes combines a range of different characteristics. The design of such items is important, as questions that contain multiple dimensions run contrary to good survey design principles. Multiple questions or vignettes that alter single characteristics provide useable information on intersectionality.

We find an example in the Eurobarometer (2015) which asks two separate questions about attitudes to lesbian and gay same-sex partnerships (intersectionality between gender and sexual orientation), while the ESS (2018) contains a set of eight questions which ask respondents' approval for different family/marital choices for men and women.

How much do you approve or disapprove if a man: gets divorced while he has children under 12 ?

How much do you approve or disapprove if a woman: gets divorced while she has children under 12 ?

Gender role attitudes questions often contain judgements about gender and family roles together. However we have included these as a separate category of questions under the gender topic rather than as examples of intersectionality. 


\section{TABLE 3.2 MOST FREQUENTLY ASKED QUESTIONS: MISCELLANEOUS EQUALITY}

\begin{tabular}{|c|c|c|c|c|c|c|c|c|c|c|c|c|c|c|c|c|c|c|c|c|c|}
\hline & YEAR: & ‘00 & ‘01 & ‘02 & ‘03 & ‘04 & ‘05 & ‘06 & ‘07 & ‘08 & ‘09 & '10 & '11 & '12 & '13 & '14 & '15 & '16 & '17 & '18 & Total \\
\hline \multirow{3}{*}{ ESS } & $\begin{array}{l}\text { Now I will briefly describe some people. Please } \\
\text { listen to each description and tell me how much } \\
\text { each person is or is not like you... }\end{array}$ & & & 1 & & 1 & & 1 & & 1 & & 1 & & 1 & & 1 & & 1 & & 1 & 9 \\
\hline & $\begin{array}{l}\text { He/She thinks it is important that every person } \\
\text { in the world should be treated equally. He/She } \\
\text { believes everyone should have equal } \\
\text { opportunities in life. }\end{array}$ & & & & & & & & & & & & & & & & & & & & \\
\hline & $\begin{array}{l}\text { Very much like me, like me, somewhat like me, a } \\
\text { little like me, not like me, not like me at all }\end{array}$ & & & & & & & & & & & & & & & & & & & & \\
\hline \multirow{4}{*}{ EB } & $\begin{array}{l}\text { Three most important values: tolerance? } \\
\text { Mentioned, Not Mentioned }\end{array}$ & & & & 1 & & & 1 & & 1 & 1 & 1 & & 1 & 1 & 1 & 1 & 1 & 1 & 1 & 12 \\
\hline & $\begin{array}{l}\text { Three most important values: equality? } \\
\text { Mentioned, not mentioned }\end{array}$ & & & & 1 & & & 1 & & 1 & 1 & 1 & & 1 & 1 & 1 & 1 & 1 & 1 & 1 & 12 \\
\hline & $\begin{array}{l}\text { Three most important values: human rights? } \\
\text { Mentioned, not mentioned }\end{array}$ & & & & 1 & & & 1 & & 1 & 1 & 1 & & 1 & 1 & 1 & 1 & 1 & 1 & 1 & 12 \\
\hline & $\begin{array}{l}\text { Three most important values: respect cultures? } \\
\text { Mentioned, not mentioned }\end{array}$ & & & & 1 & & & 1 & & 1 & 1 & 1 & & 1 & 1 & 1 & 1 & 1 & 1 & 1 & 12 \\
\hline
\end{tabular}

Source: Authors' analysis based on ESS and Eurobarometer 2000-2018. 


\section{CHAPTER 4}

\section{Discrimination}

In addition to measuring attitudes to equality among the general public, the surveys covered in this report also ask respondents whether they themselves have experienced discrimination or if they have witnessed discrimination. In each case, they ask the respondent on what ground this discrimination occurred, and these grounds have significant overlap with the grounds set out in Irish legislation. In this chapter, we provide an overview of these questions and detail the frequency with which they appear in respect of each equality group. As in Chapter 3, we also spell out in detail some discrimination questions which appear particularly frequently (five times or more) over the period.

These questions are in some respect the inverse of the attitudinal questions discussed in Chapter 3. If an equality group is viewed negatively by the general public, we would expect them to have a greater propensity to report experiencing discrimination, and we might also expect survey respondents to be more likely to report witnessing discrimination against them. Verifying consistency between public attitudes and experiences of discrimination is therefore a useful robustness check in quantitative equality research. However, this is dependent on having a sufficient sample size to capture the experiences of protected groups, which is not the case of the smaller groups in the Irish samples of cross-national European surveys (see Chapters 1 and 2). ${ }^{26}$

Similar to the questions examining attitudes, the equality groups do not have an equal number of questions each relating to experiencing or witnessing discrimination. As before, the group with the largest number of questions is 'race, ethnicity and nationality', with almost twice as many questions as the next most commonly asked about group; 'gender'. There are no questions asked about experiences of discrimination or about witnessing discrimination on the basis of civil status, family status, or being a recipient of social welfare.

26 The European Commission's Guidelines on Improving the Collection and Use of Equality Data note that 'Data collection on the discrimination grounds of racial or ethnic origin, religion or belief, sexual orientation and gender identity tends to be least developed in Member States' (2017, p.6). 


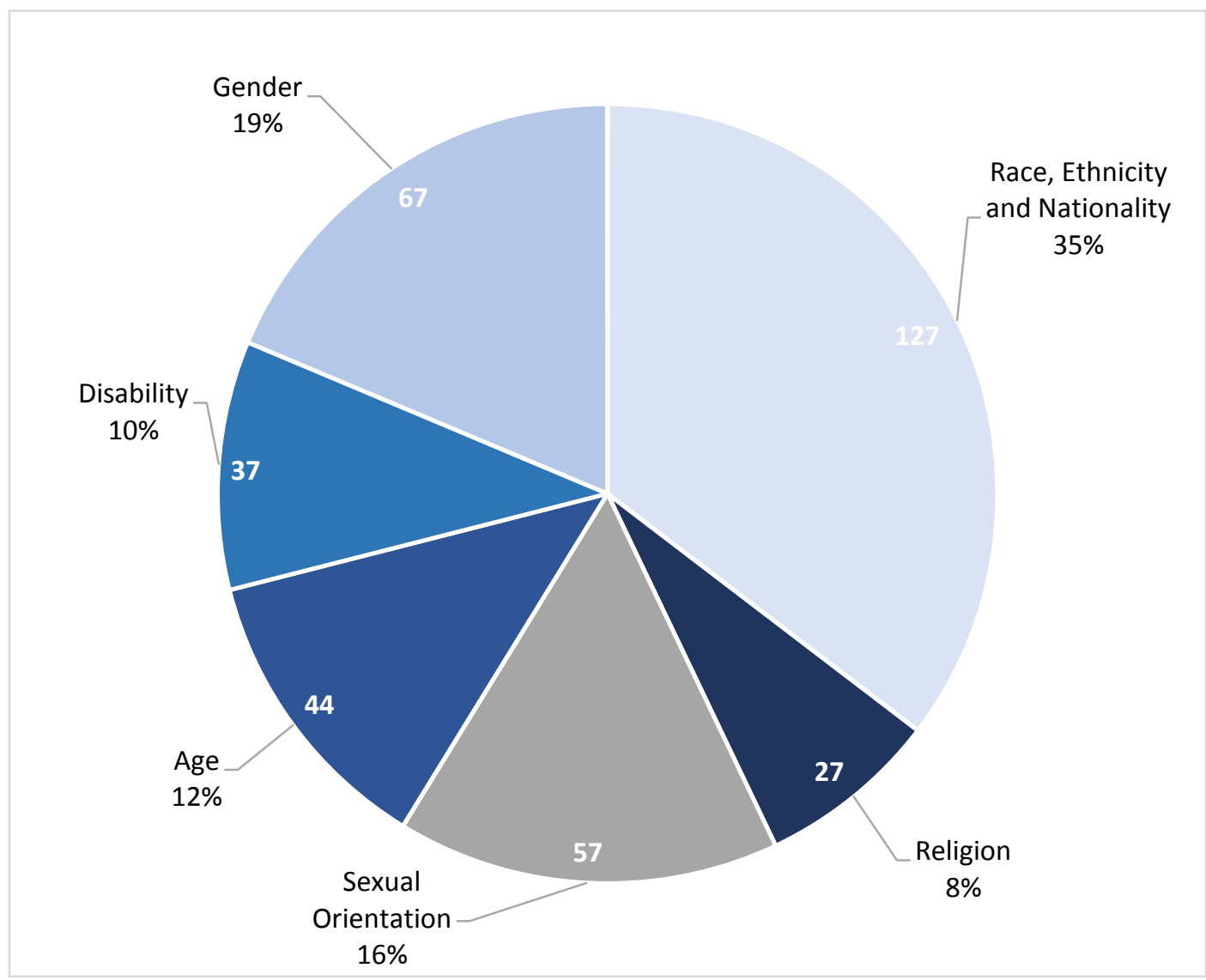

Source: Authors' analysis based on Eurobarometer, ESS, EVS, FRA and ISSP 2000-2018.

\subsection{EXPERIENCES OF DISCRIMINATION}

Questions regarding experiences of discrimination are very common, with over 300 being asked overall, and questions on this topic being asked most years. Experiences of discrimination are asked regarding most of the target groups, although there is none about experiences of discrimination relating to civil status, family status or being a recipient of social welfare payments.

Each survey approaches these questions slightly differently, and there are some inconsistencies in the application of the questions over time. Respondents are often given a timeframe in which to recall experiences of discrimination. In the Eurobarometer and ESS this is often one or two years but in the FRA surveys some questions refer to experiences in the previous five years. It is also commonplace for respondents to be asked if they had experienced discrimination in a given place or domain of life. These include the workplace, schools, housing markets and accessing public services. 
Questions in Eurobarometer relating to experiences of discrimination typically ask whether respondents have ever experienced discrimination, and if the participant answers that they have, they are asked why they think they were discriminated against. They can then choose from options relating to the different target groups (e.g. race, religion, nationality, disability etc.), as well as a residual 'other' category. In the questionnaires, discrimination is defined as 'when a person or group is treated less favourably than others because of personal characteristics'. Over the period examined, four Eurobarometer surveys of discrimination were carried out, in 2006, 2009, 2012 and $2015 .{ }^{27}$

In the ESS there are a few questions about specific discrimination experiences within the last year. For instance in 2008, respondents are asked how often people have 'shown prejudice' against them or treated them unfairly. However, the most common ESS question, which appears in each wave, asks participants if they are a member of a group that is discriminated against generally, and if so, on what grounds do they experience discrimination. No definition of discrimination or prejudice is provided in the surveys.

In the FRA surveys, almost all questions are about discrimination experiences within the past one to five years. The FRA questions also usually specify a place where the discrimination happened, such as in a shop or when looking for a job. While most surveys ask about discrimination experiences, the FRA also asks about experiences of violence or harassment due to the respondent being a member of a particular target group. The FRA defines discrimination as 'when somebody is treated less favourably than others because of a specific personal feature such as their age, gender, gender identity, sexual orientation, minority background or for any other reason' and harassment as 'unwanted and disturbing behaviour towards you such as name calling, or ridiculing that did not involve actual

27 https://ec.europa.eu/commfrontoffice/publicopinion/archives/ebs/ebs_263_en.pdf; https://ec.europa.eu/commfrontoffice/publicopinion/archives/ebs/ebs_317_en.pdf; https://ec.europa.eu/commfrontoffice/publicopinion/archives/ebs/ebs_393_en.pdf; https://ec.europa.eu/commfrontoffice/publicopinion/index.cfm/ResultDoc/download/DocumentKy/680 05 [last accessed 21 August 2019]. 
violence or the threat of violence' ${ }^{28}$ There are no questions about discrimination experiences in either the ISSP or EVS.

Questions on the experience of discrimination were asked in most years covered by our analysis, but some groups have more questions asked about them than others. The count of questions peaks in 2002, 2012 and 2016. The 2012 and 2016 spikes are due to the FRA waves that occurred in those years, relating to ethnicity, gender and sexual orientation. The spike in 2002 is mainly due to a Eurobarometer module on discrimination that occurred that year, although there are also some questions from the ESS.

\section{FIGURE 4.2 DISCRIMINATION EXPERIENCE QUESTION COUNT, ALL SURVEYS}

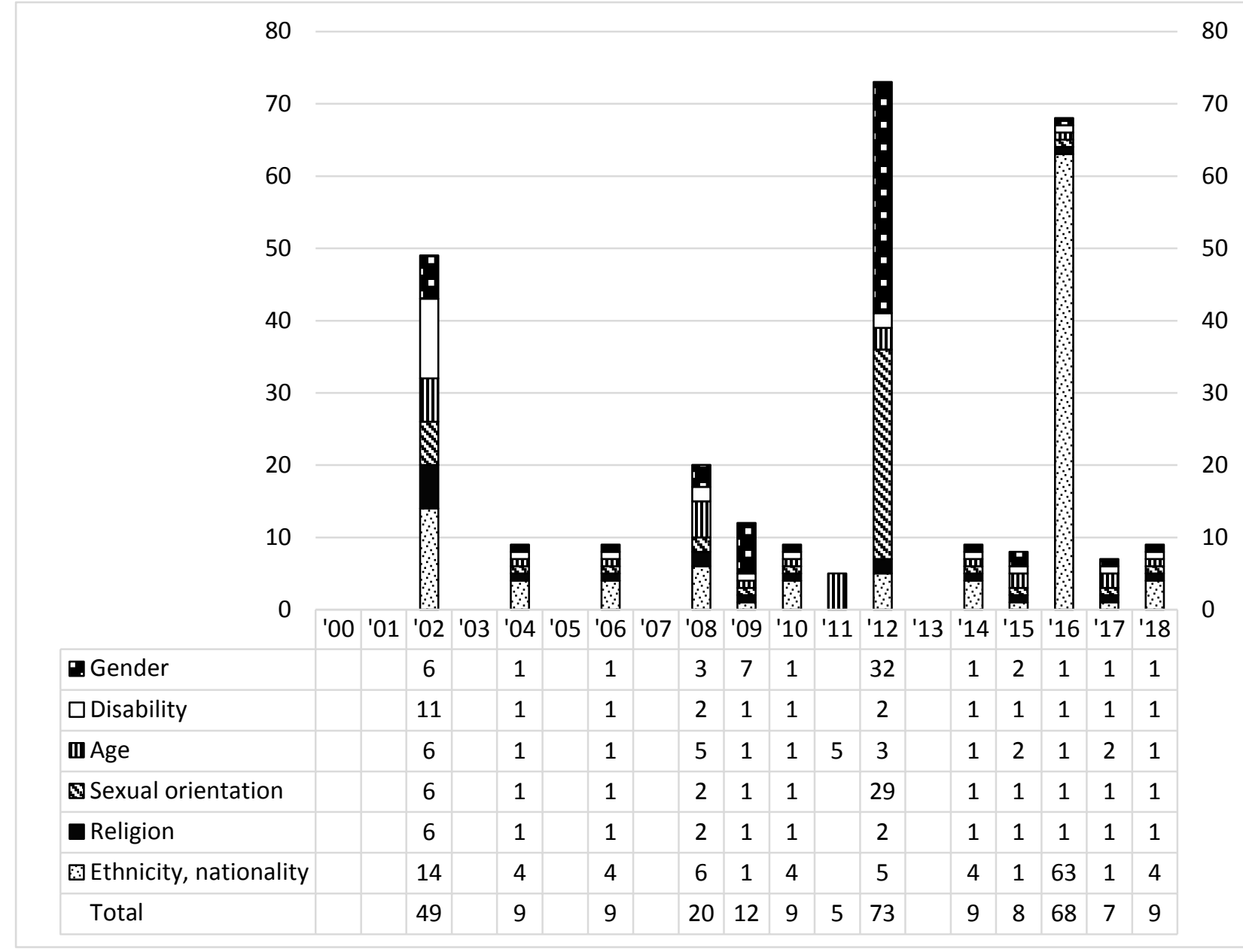

Source: Authors' analysis based on Eurobarometer, ESS, EVS, FRA and ISSP 2000-2018.

28 https://fra.europa.eu/sites/default/files/eu-lgbt-survey-technical-report_en.pdf [last accessed 8 October 2019]. 
The group with the largest number of questions asked about experiences of discrimination is the race, ethnicity and nationality group. This is partly because it is a very broad concept, incorporating questions on discrimination experienced because of ethnicity, race, language, and nationality. This group is referred to in most years that there are questions about discrimination experiences. In particular, there is a huge number of questions in 2016 about race, ethnicity and nationality. This is due to the FRA 'Second European Minorities and Discrimination Survey' which took place that year, consisting almost exclusively of questions on experiences of discrimination and violence. Questions about experiencing violence and harassment are all from this FRA survey, with the other surveys asking only about experiencing discrimination rather than violence. The questions on violence relate to various acts such as hearing offensive comments, being physically attacked or being gestured at offensively.

Questions relating to experiencing gender discrimination also appear most years. These questions are particularly common in 2012, due again to a FRA survey, this time on violence against women. All of the questions on violence or harassment experiences are from this survey, while the other surveys focus on discrimination. These questions are distinct from the questions on gender-based violence in Chapter 3 as they ask about past experiences of violence while the questions in Chapter 3 asked about attitudes towards gender-based violence in the abstract. The questions on violence ask about several types of violent acts such as psychological abuse, being forced into sexual activity, or being physically attacked. One anomalous type of question in this category is a set of questions from Eurobarometer where the respondent is asked whether they have been a victim of or witnessed gender discrimination in the last three years and the respondent can say yes to either being a victim or a witness. The other surveys, as well as most Eurobarometer modules, ask separate questions about witnessing and experiencing, rather than including them in the same question. We have (arbitrarily) included these questions in the count of questions relating to discrimination experiences, rather than witnessing (see below). This module appears in 2009 and asked about discrimination in various situations such as political life and healthcare. 
There are only three questions regarding experiences of discrimination due to being transgender. One is in the 2012 FRA LGBT survey, and two are in Eurobarometer, in 2012 and 2015. None of these questions relates to a specific place or life domain.

Survey questions about experiences of discrimination among the sexual orientation target group are more common than questions that tap attitudes towards people who identify as gay, lesbian or bisexual. This is largely due to a 2012 FRA survey on LGBT people in the European Union, although questions on discrimination due to sexual orientation appear in most years. As with the other target groups, questions about harassment and violence only appear in the FRA. There are very few questions on violence in the LGBT FRA survey, with two questions about physical attacks and two about harassment, as most of the questions focus on discrimination.

Questions on discrimination due to age are asked every year in which discrimination experience questions are fielded. These questions are the most frequently asked questions relating to age. These questions include questions on discrimination due to age, without specifying youth or old age, as well as specific questions about experiences due to being under 30 or over 55 years of age. There are no questions relating to violence directed towards someone because of their age.

Discrimination experiences due to religion and disability are the least popular categories of discrimination experience questions. These questions are only about discrimination, not violence. Many disability questions ask about disability in general, but some specifically mention physical disability and mental illness. Most questions on experiences of discrimination among people with disabilities were fielded as part of the Eurobarometer's 2002 module on 'Discrimination Issues'. A question on discrimination on the basis of religion is asked in every round of the ESS, and also features in the Eurobarometer's 2002 module. None of these questions specifies any particular religion.

While questions on experiences of discrimination are very common overall, few of them were asked five times or more. This is because these types of questions change a lot between surveys, for example, covering different time periods since the discrimination took place, different locations, and different acts such as violence or workplace discrimination. They also have differences in what responses are collected, with some being yes/no answers 
and some documenting how many times discrimination has taken place. This precludes direct comparison over time, so they were not included in the same question count. There are two questions that are very frequent, however. The first is the ESS question which asks respondents if they are a member of a group that is discriminated against, and if so, on what grounds do they experience discrimination. This is asked in each ESS wave; nine times overall. The other common question is from Eurobarometer, where respondents are asked if in the last 12 months they have felt discriminated against on the basis of various grounds such as gender or religious belief. This question appears five times overall for sexual orientation, ethnic origin and disability, but is asked fewer times for the other target groups.

\subsection{WITNESSING DISCRIMINATION}

Questions about witnessing discrimination are much less frequent than those on experiencing discrimination, with over four times as many of the latter asked in the surveys. Witnessing discrimination is only asked about in six years of surveys. Like the discrimination experience questions, there are no questions about witnessing discrimination due to family status, civil status or social welfare payments.

The questions on witnessing discrimination come almost entirely from Eurobarometer, with a few from the FRA but none from the ESS, EVS or ISSP. The Eurobarometer questions follow the same format as the questions on experiences, and are usually asked immediately before the equivalent question on experiences. They ask about witnessing discrimination either in certain places such as the workplace, and typically specify a timeframe such as the past two years. The FRA questions are about knowing someone who has been discriminated against rather than directly witnessing discrimination. It also includes some questions about knowing someone who has been physically attacked due to their race (2016) or who has been a victim of domestic violence (2012). These FRA questions are the only questions about witnessing violence, as opposed to discrimination. Most years that include questions on witnessing discrimination ask about all the target groups.

The questions about witnessing discrimination peak in number in 2002 due to an extensive Eurobarometer module on discrimination that year. There are also Eurobarometer modules in 2008 and 2009 on discrimination but they ask fewer questions about witnessing 
discrimination. The questions on witnessing age discrimination in 2011 come from the Eurobarometer module on 'active aging'. The slight increase in questions in 2012 is due to the two FRA surveys that year, as well as another Eurobarometer discrimination module. After 2012 , there are only a total of three questions asked about witnessing discrimination in all the surveys.

\section{FIGURE 4.3 WITNESSING DISCRIMINATION QUESTION COUNT, ALL SURVEYS}

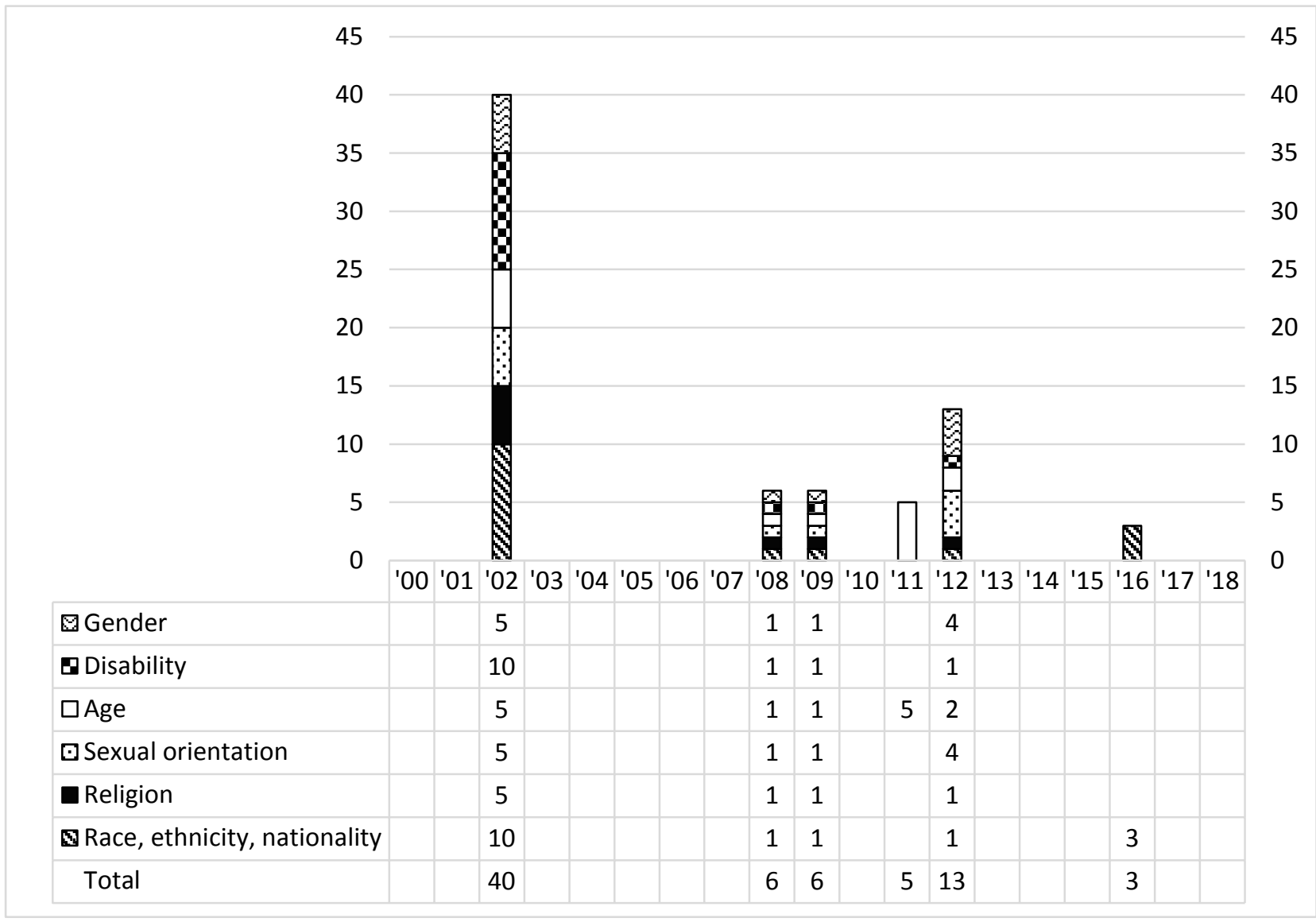

Source: Authors' analysis based on FRA and Eurobarometer 2000-2018.

In relation to race, ethnicity and nationality, Eurobarometer asks about witnessing discrimination on the basis of both ethnic origin and nationality. Only three of the questions are from the FRA survey of 2016, which ask about whether the respondent knows someone who has been called names, discriminated against or attacked due to their ethnicity or nationality in the last year. The highest number of race, ethnicity and nationality questions appear in 2002 due to a Eurobarometer module on discrimination. The only questions on witnessing discrimination after 2012 are those relating to this group, due to the 2016 FRA survey. 
Questions about witnessing discrimination due to gender are most frequent in 2012 because of the FRA surveys on gender and sexual orientation, and in 2002 because of the Eurobarometer module on discrimination. The FRA has two questions about whether the respondent knows any women who have been victims of domestic violence, which is not asked about in Eurobarometer.

There is one question about witnessing discrimination against transgender people in the 2012 Eurobarometer module on discrimination.

Witnessing age discrimination is inquired about in most years with discrimination witness questions. It is also the only type of question on witnessing discrimination asked in 2011 which is fielded as part of the Eurobarometer module on 'active aging'. Most of the questions just ask about the broad topic of discrimination on the basis of age, but two in 2012 specify witnessing age discrimination against those under 30 or over 55 years of age. Questions about witnessing age discrimination are only asked in Eurobarometer.

The questions on disability and religion are similar to those about experiencing discrimination as a member of those target groups. The religion questions do not specify any religion, and in 2002 there are questions about witnessing discrimination on the basis of 'mental illness', which is not asked in later surveys. Witnessing religious or disability discrimination is only asked about in Eurobarometer, with the majority of the questions coming from the 2002 discrimination module.

No question about witnessing discrimination appears more than five times overall. 



\section{CHAPTER 5}

\section{Conclusions}

\subsection{OVERVIEW}

This report has mapped out the data available on attitudes to equality and human rights issues in Ireland collected in European surveys between 2000 and 2018. These surveys provide a rich reservoir of evidence that is relatively untapped. The intention of this exercise is to provide information on the types of data available, including the groups and issues covered, that can be used to help inform policy development and research.

The research team searched the questionnaires of over 125 attitude surveys fielded during the period 2000 to 2018 for relevant questions. The search was limited to surveys that: were conducted cross-nationally, including in Ireland; assess attitudes to equality and human rights; are methodologically robust; and are potentially available to researchers and policymakers for analysis. These criteria exclude surveys that are conducted by private polling agencies or marketing companies.

The search identified 1,509 questions from five sets of surveys: the Eurobarometer, the European Social Survey (ESS), the European Values Study (EVS), the International Social Survey Programme (ISSP) and the surveys of the EU Fundamental Rights Agency (FRA). The questions are subsequently categorised according to the equality groups and the themes addressed. This analysis of question content and coverage allows us to identify opportunities and gaps for research on attitudes to equality and human rights in Ireland.

The report and the accompanying Excel datasheet contribute to the goal of improving the accessibility and use of equality data as outlined in the EU Guidelines on Improving the Collection and Use of Equality Data, by making it easier for policymakers and researchers to find existing relevant data on attitudes to equality and human rights in comparative European surveys over the last two decades. 
Carefully designed surveys based on representative samples that investigate the attitudes of the overall population to protected groups can provide an important piece of the picture on equality and discrimination in Ireland. They can provide, for example, information on the perceived social distance between groups and the extent to which respondents are tolerant of diversity or the prevalence of stereotypical views (for example McGinnity et al., 2018a). Such data complement other sources of information on inequalities and discrimination such as data on outcomes and from experimental studies (McGinnity et al., 2018b). Attitudinal surveys can also help discern the level of public support for social policies aimed at reducing inequalities. They can also help understand the level of support for the protection of human rights and identify areas where awareness or information campaigns might be useful. The cross-national nature of the surveys examined means that such data can also provide evidence on how attitudes in Ireland compare to those elsewhere in Europe, while the repetition of questions over time can provide insights into how attitudes and people's experiences have changed over time.

Data on the subjective experience of discrimination can also add to evidence from objective sources to help understand the extent and impact of unfair treatment. With the exception of the FRA surveys which focus on particular vulnerable groups, the European surveys do not include sufficient numbers in most sub-sample groups to investigate the experience of many minority groups. However, these surveys can show differences between bigger groups, e.g. by sex or age category, and highlight the prevalence of perceived discrimination in different arenas such as at work or accessing services. Moreover, questions on witnessing discrimination can potentially be answered by all respondents and therefore will not suffer from the small number problem.

Data that allow the disaggregation of attitudes or discrimination experiences by smaller minority groups would require larger samples than are typically collected in cross-national social surveys. Given the scale of effort, co-ordination and funding needed to field surveys cross-nationally, it is unlikely that the sample numbers will be scaled up sufficiently to capture small groups of the population. One option would be to fund booster samples of populations of interest within a national setting. Alternatively, attitudinal questions could be added to existing large scale national surveys, such as the Labour Force Survey (LFS). Questions on discrimination experience in Ireland have recently moved from the LFS to a 
new General Household Survey; however, the size of the sample is much smaller (CSO, 2019), which means that experiences of certain protected groups will not be captured.

It should also be noted that it may be difficult for the observer or indeed the victim to ascertain the motive behind discriminatory action. The strengths and limitations of selfreports of discrimination are discussed in McGinnity et al. (2017 and 2012) and researchers advise the triangulation of evidence from different sources and using different methods to assess discrimination and unfair treatment (Pager and Shepherd, 2008; Blank et al., 2004; Heath et al., 2013).

\subsection{DATA OPPORTUNITIES AND GAPS}

Our analysis shows that the availability of survey data is uneven across equality groups and also differs over time. The equality group most consistently covered in the attitudes questions is nationality/race/ethnicity. Questions on attitudes towards individuals of different ethnicity/nationality/race or to migration in general are available in all but one of the 19 years examined. In total, 449 questions on race, ethnicity and nationality were identified.

Attitudes to social welfare recipients are grouped together with attitudes to the role of social welfare, which was the next most common category. Such questions test general support for government redistribution of resources and whether respondents believe in individualistic or societal/structural causes of poverty and inequality. These attitudes are relevant to support for social and economic rights. Overall there were 221 such questions, which reflects the breadth of this category.

Questions relating to gender/gender roles are also frequently included in attitudinal surveys, with 226 such questions identified. These three groups accounted for almost threequarters of the questions with the other categories much less frequently represented. Attitudes towards people with disabilities appeared 62 times, questions on age groups 57 times, and the LGB community 43 times. 
Attitudes towards religious minorities was the topic least covered $(\mathrm{N}=31)$. Questions relating to attitudes to civil and family status were also uncommon with only 37 such questions identified over the 19-year period examined.

Trends over time also vary across protected groups. Few questions on attitudes to the LGB community were identified in the first half of the period; however, this topic has become increasingly well covered over time. This is also true of questions relating to transgender people, which are first included in 2012. Conversely, ageism and age-related stereotypes was a more common theme in the first half of the period but has virtually disappeared as a topic in the surveys examined since 2010. A similar downward trend is noticeable for attitudes towards people with a disability; only 16 questions have appeared since 2003.

In terms of the themes addressed, the European questionnaires analysed provide scope for investigating a range of topics including social distance/contact, tolerance of diversity and policy responses. Analysis of such questions can provide insights into where policy efforts to promote understanding and reduce prejudice might best be targeted and into the design of such policies. It is also useful to highlight where policy may encounter public resistance or support.

Interventions to reduce discrimination and protect human rights can also be guided by analysis of the questions that directly assess experience of discrimination that are outlined in Chapter 4. As is the case with questions on attitudes towards groups, questions on discrimination (experienced or witnessed) on the grounds of nationality/ethnicity/race are most common $(\mathrm{N}=127)$, followed by gender $(\mathrm{N}=67)$. However unlike the attitude questions, questions relating to discrimination on the basis of sexual orientation are relatively common $(\mathrm{N}=57)$ and age discrimination is also well represented $(\mathrm{N}=44)$. Religious discrimination is less well covered $(\mathrm{N}=27)$ and no questions were found on experiencing/witnessing discrimination on the basis of social welfare receipt, social class or other dimensions of socio-economic status.

\subsubsection{Data gaps}

Our analysis suggests there is a noticeable gap in knowledge at European level on attitudes towards those with a disability, religious minorities and younger/older age groups. There is 
also an absence of questions on social distance with reference to people in receipt of social welfare/different social class and, relatedly, questions that examine experiences of discrimination on the basis of socio-economic background. As noted above, the sample size per country means that discrimination experiences of smaller groups, such as LGBT individuals and ethnic minorities including Travellers, are unlikely to be sufficiently represented for statistical analysis in the majority of the surveys considered.

Our scrutiny of the question wording also shows that relatively few of the questions distinguish between different types of disability. Attitudes towards those with an intellectual disability are particularly poorly covered. While some of this gap on disability attitudes is addressed at the national level through surveys carried out by the National Disability Authority $(2011 ; 2017)$, this does not allow for cross-national analysis.

Questions that address intersectionality are also rare. We identified only ten such questions in the surveys that we searched. Therefore there is considerable scope to develop further survey items that investigate intersectionality which could be fielded in European or national surveys.

There are also few questions that directly assess respondents' views of the protection of human rights and the importance they place on this relative to other issues.

These gaps suggest the need for future European data collection on attitudinal questions on human rights more broadly, intersectionality, and questions relating to those with disabilities, religious minorities and those from different socio-economic backgrounds. The latter group might be distinguished on the basis of social class background, residential area and labour market status but its incorporation would support efforts to promote socioeconomic rights and to improve social mobility in European societies. The decline in attitudinal questions on age groups/ageism highlights a need for future data collection to support policy development in the context of an ageing population across Europe.

\subsubsection{Trend data}

Frequent replication of questions (five or more times) is relatively uncommon, and is mostly confined to core questions in the European Social Survey. This is partly due to the length of 
the period covered in our analysis - 19 years - which means that five repeats involves very regular repetition. Given that international comparative data are costly to collect it is not surprising that such frequent repetition is rare. The primary strength of these surveys is their comparability across countries rather than over time. Nevertheless, many more questions are repeated more than once, with longer intervals in between. The database constructed for this report, which is available at https://www.esri.ie/publications/europeansurvey-data-on-attitudes-to-equality-groups-and-human-rights and https://www.ihrec.ie/documents/european-survey-data-on-attitudes/, can also be used to identify any questions repeated over the period.

\subsubsection{Lessons for questionnaire design}

The questionnaires examined for this survey provide a repository of questions that might be used by researchers or policymakers interested in collecting their own information.

Questions included in international surveys have already been tested in the field, though the quality/validity is likely to vary from survey to survey and for individual questions. The ESS in particular has a transparent and rigorous approach to survey development. Documents outlining the process of question development including the quality control measures and a description of the conceptual basis for the survey questions are available online. ${ }^{29}$ There is also extensive ongoing testing of question wording and translation (see Jowell et al., 2007). This provides an example of best practice in questionnaire design.

The availability of the microdata allows researchers themselves to test whether individual question items generated sufficient response variation among respondents in Ireland, to examine item non-response, and to test validity by examining the relationship between the responses and other measures to see if they are in the expected direction. ${ }^{30}$ The current exercise has focused on developing a question bank. We have not analysed the data produced to check for validity, reliability and representativeness; therefore inclusion in the question bank is not an endorsement of data quality.

29 https://www.europeansocialsurvey.org/methodology/ess_methodology/source_questionnaire/source_ questionnaire_development.html. Also see question module design templates on the ESS website. 
While the questions included in the analysis and database have already been fielded by international agencies, there are, nevertheless, lessons to be learned for improving questionnaire design on equality attitudes. The distinction between normative opinions (how things should be or what outcome is favoured) and more observational questions (perceptions of how things are) which may be objectively verifiable, was not always clear in the questions. For example, in the year 2000 the Eurobarometer poses the following statement to participants; 'In order to be fully accepted members of our society, people belonging to minority groups must give up their own culture'. It is not clear from this question whether the respondent is being asked about their opinion, i.e. whether minority groups should give up their culture, or whether the prevailing social or political forces mean that they do need to give up their culture, regardless of whether the respondent likes this fact or not. If this question is intended to be posed normatively, it should be clear that the respondent is being asked for his or her opinion. If the surveyor is interested in the respondent's insights into the fact of the matter, the question could be prefaced with a qualification such as 'regardless of your view on the matter, is it the case that today in Ireland minorities need to give up their culture to be fully accepted members of society'.

Being clear of the concepts or issue that the question is designed to address should help avoid such ambiguity in question wording.

Social research on self-reported discrimination suggests that it is best practice to provide respondents with a definition of what constitutes discrimination (see McGinnity et al., 2012 for discussion). This is done in the FRA surveys and the Eurobarometer but not in the ESS.

The potential for social desirability in survey responses was outlined in Chapter 1 (see also Krumpal, 2013). Attitudes on equality and human rights may be particularly prone to such bias; for example attitudes to ethnic minorities and immigration (Janus, 2010), which may lead to an under-estimation of prejudice. Careful question design can help to mitigate such problems. Questions need to be phrased in a way that ensures a variety of answers (e.g. agreement and disagreement) are possible and respondents are not led in one direction. The introductions to questions, response categories and question ordering are also important in order to minimise priming respondents so they are more likely to pick one 
response over another, and to avoid any implicit suggestion that one response is more socially accepted than others.

Despite these limitations, well designed questions that are carefully harmonised across countries and that are administered through surveys applying the best scientific practices in terms of sampling procedures, interviewing, weighting, etc. are an invaluable resource for researchers and policymakers in Ireland. Such data not only provide insights into the situation in Ireland but also provide the international context which can inform efforts to promote equality and human rights. 


\section{REFERENCES}

Albarracin, D., M.P. Zanna, B.T. Johnson and G.T. Kamkale (2005). 'Attitudes: Introduction and Scope', in The Handbook of Attitudes, NY: Psychology Press.

Blank, R.M., M. Dabady and C.F. Citro (Eds.) (2004). Measuring racial discrimination. Washington DC: The National Academies Press.

Bond, L., F. McGinnity and H. Russell (Eds.) (2010). Making Equality Count Irish and International Research Measuring Equality and Discrimination, Dublin: The Liffey Press.

Brownback, A. and A. Novotny (2018). 'Social desirability bias and polling errors in the 2016 presidential election', Journal of Behavioral and Experimental Economics, Vol. 74, pp. 38-56.

CSO (2019). Equality and Discrimination, CSO statistical release, 04 July 2019.

Eagly, A.H. and S. Chaiken (1993). The Psychology of Attitudes, Florida: Harcourt Brace Jovanovich.

European Commission (2018). 'High Level Group on Non-discrimination, Equality and Diversity', Guidelines on Improving the Collection and Use of Equality Data, European Commission.

European Social Survey (2018). 2016 Documentation Report. Available at: www.europeansocialsurvey.org/docs/round8/survey/ESS8_data_documentation _report_e02_1.pdf (Accessed August 2019).

European Social Survey (n.d.). 'ESS Methodology'. Available at: www.europeansocialsurvey.org/methodology/ess_methodology (Accessed August 2019).

European Union Agency for Fundamental Rights (2009). EU-MIDIS Technical Report: Methodology, Sampling and Fieldwork. Available at: https://fra.europa.eu/sites/default/files/eu-midis_technical_report.pdf

European Union Agency for Fundamental Rights (2013). EU LGBT Survey Technical Report. Available at: fra.europa.eu/sites/default/files/eu-lgbt-survey-technicalreport_en.pdf (Accessed August 2019).

European Union Agency for Fundamental Rights (2014). Violence Against Women: An EU Wide Survey - Survey methodology, sample and fieldwork. Luxembourg: Publications Office of the European Union.

European Union Agency for Fundamental Rights (2017). Second European Union Minorities and Discrimination Survey Technical Report. Luxembourg: Publications Office of the European Union. 
European Values Survey and GESIS Data Archive for the Social Sciences (2016). EVS 2008 Method Report. Köln: GESIS - Leibniz-Institut für Sozialwissenschaften.

GESIS (n.d.). 'ISSP - The International Social Survey Programme'. Available at: zacat.gesis.org/webview/index.jsp?mode=documentation\&submode=catalog\&ca talog=http://zacat.gesis.org/obj/fCatalog/Catalog6 (Accessed August 2019).

GESIS (n.d.). Standard and Special Topics Eurobarometer. Available at: $\quad$ www.gesis.org/eurobarometer-data-service/survey-series/standard-special-eb (Accessed August 2019).

Heath, A., T. Liebig and P. Simon (2013). 'Discrimination against Immigrants - Measurement, Incidence and Policy Instruments' in OECD (Eds.) International Migration Outlook 2013. Paris: OECD Publishing.

Heerwegh, D. (2009). 'Mode Differences Between Face-to-Face and Web Surveys: An Experimental Investigation of Data Quality and Social Desirability Effects', International Journal of Public Opinion Research 21:111-121.

IHREC (2017). Observations on the Equality (Miscellaneous Provisions) Bill 2017, Dublin: IHREC.

IHREC (2019). Strategy Statement 2019-2021, Dublin: Irish Human Rights and Equality Commission.

Inglehart R. (1977). The Silent Revolution: Changing Values and Political Styles Among Western Publics. Princeton, NJ: Princeton University Press.

Inglehart R. (1997). Modernization and Postmodernization: Cultural, Economic, and Political Change in 43 Societies. Princeton, NJ: Princeton University Press.

Janus (2010). 'The Influence of Social Desirability Pressures on Expressed Immigration Attitudes', Social Science Quarterly, 91(4), pp. 925-946.

Jowell, R., C. Roberts, R. Fitzgerald and G. Eva (Eds.) (2007). Measuring Attitudes CrossNationally: Lessons from the European Social Survey. London: Sage Publications.

Krumpal, I. (2013). 'Determinants of Social Desirability Bias in Sensitive Surveys: A Literature Review', Quality and Quantity, Vol. 47, pp. 2025-2047.

McGinnity, F., D. Watson and G. Kingston (2012). Analysing the experience of discrimination in Ireland; Evidence from the QNHS Equality Module 2010, Dublin: Equality Authority and ESRI.

McGinnity, F., R. Grotti, H. Russell and E. Fahey (2018a). Attitudes to Diversity in Ireland. Dublin: ESRI/IHREC, Research Series.

McGinnity, F., R. Grotti, O. Kenny and H. Russell (2017). Who experiences discrimination: evidence from the QNHS Equality Modules. Dublin: ESRI/IHREC.

McGinnity, F., R. Grotti, S. Groarke and S. Coughlan (2018b). Ethnicity and Nationality in the Irish Labour Market. ESRI/IHREC, Research Series. 
NDA (2011). A national survey of public attitudes to disability in Ireland. Dublin: National Disability Authority.

NDA (2017). A national survey of public attitudes to disability in Ireland. Dublin: National Disability Authority.

Pager, D. and H. Shepherd (2008). 'The sociology of discrimination: racial discrimination in employment, housing, credit, and consumer markets', Annual Review of Sociology Vol. 34, pp. 181-209.

Simmons, R. (2001). 'Questionnaires' in N. Gilbert (Ed.) Researching Social Life. London: Sage.

The International Social Survey Programme (n.d.). 'The International Social Survey Programme'. Available at: w.issp.org/about-issp (Accessed August 2019).

United Nations (2018). A Human Rights-Based Approach to Data, UN Human Rights Office of the High Commission.

Zaller, J. and S. Feldman (1992). 'A Simple Theory of the Survey Response: Answering Questions versus Revealing Preferences', American Journal of Political Science, Vol. 36(3), pp. 579-616. 



\section{Appendix}

TABLE A.1 TOPIC CATEGORIES USED IN QUESTION-BANK SPREADSHEET

\begin{tabular}{|c|c|}
\hline & Miscellaneous \\
\hline 100 & Important values \\
\hline 102 & Human rights (importance attached to) \\
\hline 103 & Anti-discrimination action \\
\hline 104 & Equality policy \\
\hline \multicolumn{2}{|c|}{ Race, Ethnicity and Nationality } \\
\hline 201 & Social distance/contact \\
\hline 202 & Immigrants' rights (policy) \\
\hline 203 & Immigrant cultural assimilation \\
\hline 204 & Effect on country (beliefs) \\
\hline 205 & Important for being Irish \\
\hline 206 & Illegal immigration \\
\hline 207 & Views on allowing further immigration/deportation \\
\hline 208 & Views on refugees \\
\hline 209 & Representation of ethnic minorities in the media \\
\hline \multicolumn{2}{|c|}{ Religion } \\
\hline 301 & Social distance/contact \\
\hline 302 & Tolerance towards religions/religious groups \\
\hline \multicolumn{2}{|c|}{ Sexual Orientation } \\
\hline 401 & Tolerance \\
\hline 402 & Social distance/contact \\
\hline 403 & Views on LGBT parents + marriage \\
\hline \multicolumn{2}{|l|}{ Age } \\
\hline 501 & Social distance/contact \\
\hline 502 & Tolerance towards older workers \\
\hline 503 & Equality based on age \\
\hline 504 & Beliefs about young people \\
\hline 505 & Beliefs about older people \\
\hline 506 & Government responsibility for older people \\
\hline \multicolumn{2}{|c|}{ Disability } \\
\hline 601 & Social distance/contact \\
\hline 602 & Government policies regarding disability \\
\hline 603 & Tolerance towards people with a disability \\
\hline \multicolumn{2}{|l|}{ Gender } \\
\hline 701 & $\begin{array}{l}\text { Perceived obstacles to gender equality in positions of } \\
\text { power }\end{array}$ \\
\hline 702 & Views on gender equality \\
\hline 703 & Social distance gender \\
\hline 704 & Gender roles \\
\hline 705 & Views on gender based violence \\
\hline 706 & Transgender questions \\
\hline
\end{tabular}


TABLE A.1 CONTD.

\begin{tabular}{|c|l|}
\hline $\begin{array}{c}\text { Civil and family status } \\
\mathbf{8 0 1}\end{array}$ & \multicolumn{1}{c}{ Miscellaneous Contd } \\
\hline $\mathbf{8 0 2}$ & Lone parents \\
\hline $\mathbf{8 0 3}$ & Family/civil status tolerance \\
\hline $\mathbf{8 0 4}$ & Views on premarital sex/living together/having children \\
\hline General Equality & Family policy \\
\hline $\mathbf{9 0 1}$ & \\
\hline $\mathbf{9 0 2}$ & Why are people poor/homeless \\
\hline $\mathbf{9 0 3}$ & Government responsibility for poverty \\
\hline $\mathbf{9 0 4}$ & Views on equality of income \\
\hline Experience discrimination & Views on unemployment/social welfare \\
\hline E2 & Experience discrimination: race, ethnicity nationality \\
\hline E3 & Experience discrimination: religion \\
\hline E4 & Experience discrimination: sexual orientation \\
\hline E5 & Experience discrimination: age \\
\hline E6 & Experience discrimination: disability \\
\hline E7 & Experience violence/discrimination: gender \\
\hline Witness discrimination & \\
\hline W2 & Witness discrimination: race, ethnicity nationality \\
\hline W3 & Witness discrimination: religion \\
\hline W4 & Witness discrimination: sexual orientation \\
\hline W5 & Witness discrimination: age \\
\hline W6 & Witness discrimination: disability \\
\hline W7 & Witness violence/discrimination: gender \\
\hline
\end{tabular}


16-22 Sráid na Faiche,

Baile Átha Cliath 7, D07 CR20 16-22 Green Street,

Dublin 7, D07 CR20

Guthán/Phone + 353 (0) 18589601

Ríomhphost/Email info@ihrec.ie

Idirlíon/Web www.ihrec.ie
Whitaker Square,

Sir John Rogerson's Quay Dublin 2

Phone +35318632000

Fax +35318632100

Email admin@esri.ie

Web www.esri.ie 